Research Paper

\title{
G6PD upregulates Cyclin E1 and MMP9 to promote clear cell renal cell carcinoma progression
}

\author{
Qiao Zhang ${ }^{*}$, Yueli Ni1 ${ }^{*}$, Shujie Wang2\#*, Yannick Luther Agbana1, Qiaoqiao Han ${ }^{1}$, Wenjing Liu², \\ Honggang Bai ${ }^{1,3}$, Zihan $\mathrm{Yi}^{4}$, Xiaojia Yi ${ }^{1}$, Yuzhi Zhu ${ }^{1}$, Buqing Sai ${ }^{1}$, Lijuan Yang ${ }^{1}$, Qiong Shi ${ }^{5}$, Yingmin \\ Kuang6, Zhe Yang ${ }^{\circledR}$, Yuechun Zhu ${ }^{\circledR}$ \\ 1. Department of Biochemistry and Molecular Biology, School of Basic Medical Sciences, Kunming Medical University, Yunnan, Kunming 650500, P.R. China. \\ 2. Departments of Pathology, The First Affiliated Hospital of Kunming Medical University, Yunnan, Kunming 650032, P.R. China. \\ 3. Department of Clinical Laboratory, The Second Hospital of Jingzhou, Jingzhou, Hubei 434000, P.R. China. \\ 4. Department of Medical Oncology, The Third Affiliated Hospital of Kunming Medical University (Tumor Hospital of Yunnan Province), Yunnan, Kunming \\ 650118, P.R. China. \\ 5. Department of Clinical Laboratory, The Third Affiliated Hospital of Kunming Medical University (Tumor Hospital of Yunnan Province), Yunnan, Kunming \\ 650118, P.R. China. \\ 6. Departments of Organ Transplantation, The First Affiliated Hospital of Kunming Medical University, Yunnan, Kunming 650032, P.R. China.
}

\# Current address: Department of Biochemistry and Biophysics, School of Basic Medical Sciences, Beijing Key Laboratory of Protein Posttranslational Modifications and Cell Function, Peking University Health Science Center, Beijing 100191, P.R. China.

* These authors contributed equally.

$\square$ Corresponding authors: Professor Yuechun Zhu, Department of Biochemistry and Molecular Biology, School of Basic Medical Sciences, Kunming Medical University, 1168 Yuhua Road, Chenggong, Yunnan, Kunming 650500, P.R. China. E-mail: zhuyuechun20091119@163.com; Professor Zhe Yang, Departments of Pathology, The First Affiliated Hospital of Kunming Medical University, Yunnan, Kunming 650032, P.R. China. E-mail: zyangpku@163.com

() The author(s). This is an open access article distributed under the terms of the Creative Commons Attribution License (https://creativecommons.org/licenses/by/4.0/). See http://ivyspring.com/terms for full terms and conditions.

Received: 2021.02.01; Accepted: 2021.10.19; Published: 2022.01.01

\begin{abstract}
Background: Clear cell renal cell carcinoma (ccRCC) is a cell metabolic disease with high metastasis rate and poor prognosis. Our previous studies demonstrate that glucose-6-phosphate dehydrogenase (G6PD), the first and rate-limiting enzyme of the pentose phosphate pathway, is highly expressed in ccRCC and predicts poor outcomes of $c \mathrm{RCC}$ patients. The aims of this study were to confirm the oncogenic role of G6PD in ccRCC and unravels novel mechanisms involving Cyclin El and MMP9 in G6PD-mediated ccRCC progression.

Methods: Real-time RT-PCR, Western blot and immunohistochemistry were used to determine the expression patterns of G6PD, Cyclin El and MMP9 in ccRCC. TCGA dataset mining was used to identify Cyclin E1 and MMP9 correlations with G6PD expression, relationships between clinicopathological characteristics of $c c R C C$ and the genes of interest, as well as the prognosis of $c c R C C$ patients. The role of G6PD in ccRCC progression and the regulatory effect of G6PD on Cyclin El and MMP9 expression were investigated by using a series of cytological function assays in vitro. To verify this mechanism in vivo, xenografted mice models were established.

Results: G6PD, Cyclin El and MMP9 were overexpressed and positively correlated in ccRCC, and they were associated with poor prognosis of ccRCC patients. Moreover, G6PD changed cell cycle dynamics, facilitated cells proliferation, promoted migration in vitro, and enhanced $\mathrm{ccRCC}$ development in vivo, more likely through enhancing Cyclin El and MMP9 expression.

Conclusion: These findings present G6PD, Cyclin El and MMP9, which contribute to ccRCC progression, as novel biomarkers and potential therapeutic targets for ccRCC treatment.
\end{abstract}

Key words: ccRCC, G6PD, Cyclin E1, MMP9, proliferation, migration

\section{Introduction}

Clear cell renal cell carcinoma (ccRCC) is the most common and dangerous malignancy subtype derived from kidney tissue, accounting for approximately $80 \%$ of all renal cell carcinoma cases [1, 
2]. Globally, about 400,000 new diagnosed cases and 139,000 death cases are expected to occur per year [3]. Accumulating evidences indicate that ccRCC is a cell metabolic disease with high metastasis rate, drug resistance and poor prognosis [4,5]. Over the last decades, although some patients with ccRCC can be diagnosed at early stages and cured by surgical resection, considerable number of ccRCC patients are still confronted with unfavorable prognosis because of high recurrence rate after surgical resection, and neither chemotherapy nor radiation therapy is effective for the patients with metastases $[1,6]$. Therefore, identifying key factors which are potentially recognized as diagnostic and prognostic biomarkers and functionally involved in ccRCC progression is still of great importance and may provide efficient diagnostic and therapeutic strategies for ccRCC patients.

Glucose-6-phosphate dehydrogenase (G6PD), the first and rate-limiting enzyme of the pentose phosphate pathway, is highly expressed in certain types of tumor, including lung cancer, breast carcinoma and RCC [7-9]. It is the cornerstone of the metabolic reprogramming process in tumor cells that result in the increased production of building blocks necessary for nucleotides and lipids synthesis [10, 11]. Previous studies from our research group demonstrate that G6PD overexpression is positively associated with ccRCC development and represents a potential prognostic factor for poor outcomes in ccRCC patients [9]. Moreover, G6PD was found to promote ccRCC cell proliferation and invasion through upregulating the expression of Cyclin D1 and MMP2, respectively $[9,12]$. However, the molecular mechanisms underlying G6PD-mediated ccRCC development is not completely delineated.

Cell cycle regulatory factors are implicated in various stages of tumorigenesis [13], and aberrant expression of the molecules that regulate the G1/S phases transition has been observed in different types of malignancies, including RCC [14], implying that cell cycle defects are linked to the activation of oncogenes. However, the presence and underlying mechanisms of aberrate G1/S regulatory molecules have only partly been clarified in ccRCC. Cyclin D1 and Cyclin E1 are two crucial G1/S transition regulatory factors that are often deregulated and play oncogenic roles in tumor proliferation and progression [14]. It has been reported that Cyclin D1 is abnormally highly expressed in ccRCC and promotes cell proliferation by regulating cell G1/S transition $[14,15]$. Similarly, the protein expression of Cyclin E1 has also been reported to be higher in RCC and associated with RCC tumor behavior. High Cyclin E1 level is positively correlated with RCC aneuploidy, staging and nuclear grade. There is also an association between Cyclin E1 and the S-phase fraction and high levels of Cyclin E1 is positively associated with rapid RCC proliferation [14].

Metastasis is a complex process that involve the participation of different key genes. Extracellular matrix (ECM) remodeling is crucial for the cell adherence at the initiation of the tumor metastatic stage and matrix metalloproteinases (MMPs) are strongly implicated in the degradation of the ECM, emphasizing their crucial roles in tumor metastasis [16]. MMPs expression and activity are upregulated in certain carcinomas where they exert important roles in cancer metastasis. Especially, MMP2 and MMP9, members of the MMPs, have been found to be significantly overexpressed in RCC and involved in RCC metastasis and angiogenesis [17-19].

To the best of our knowledge, the molecular mechanism of G6PD regulating Cyclin E1 and MMP9 involvement in ccRCC progression has not yet been untangled. Here, we uncover the clinicopathological implications of G6PD, Cyclin E1 and MMP9 in ccRCC. Hence, functional and mechanistic analyses help to unravel a novel mechanism of G6PD-mediated ccRCC progression. In addition, Cyclin E1 and MMP9 show more potential implication in ccRCC progression than Cyclin D1 and MMP2 respectively.

\section{Materials and Methods}

\section{Human specimens and immunological histological chemistry (IHC) analysis}

A total of 20 pairs of ccRCC tumor specimens and matched adjacent normal tissues were obtained from ccRCC patients without any treatment before surgery at the Department of Organ Transplantation of the First Affiliated Hospital of Kunming Medical University. The obtained specimens were sectioned, embedded in paraffin at the Department of Pathology of the First Affiliated Hospital of Kunming Medical University and then used for IHC analysis. Informed consent was obtained from the patients and the study was approved by the Ethics Committee of Kunming Medical University, according to the regulations of the Declaration of Helsinki.

For IHC analysis, the paraffin-embedded tissue sections were firstly dewaxed. Next, endogenous peroxidase was removed by $3 \% \mathrm{H}_{2} \mathrm{O}_{2}$ for $10 \mathrm{~min}$ at room temperature. IHC was conducted by using General-purpose two-step detection kit (PV-9000, ZSGB-BIO, Beijing, China) according to the manufacturer's protocol. The following antibodies were used: G6PD antibody (ab133525, Abcam), Cyclin E1 antibody (bsm-52048R, Bioss, Beijing, China), MMP9 antibody (ab76003, Abcam). At last, tissues 
were stained by using $\mathrm{DAB}$ detection kit (Amplifier polymer) (DAB-2031, MXB Biotechnology, Fuzhou, China) for an appropriate time, dehydrated, mounted and photographed. The staining score which ranged from 0-12 points was calculated by the staining intensity multiplied by the percentage of stained cells as described before $[9,12]$. Staining intensity could be classified into negative ( 0 point), weak ( 1 point), moderate ( 2 points) and strong ( 3 points). Percentage of positive stained cells was designated into four types: 1 point as $<25 \%, 2$ points as $26 \sim 50 \%, 3$ points as $51 \sim 75 \%$ and 4 points as $>75 \%$. Final staining scores over 4 points were considered high expression.

\section{TCGA data analysis}

Using $\mathrm{R}$ software to login The Cancer Genome Atlas database. The transcriptome data from 535 clear cell renal cell carcinoma cases and 72 normal kidney tissues (Kidney renal clear cell carcinoma [KIRC]) were harvested and the clinicopathological information of patients were matched, of which only 528 patients had expression profile data and prognostic survival information, including survival time and survival status. We used KIRC gene expression profile data (Fragments Per Kilobase of transcript per Million fragments mapped, FPKM) for gene expression differences analysis. The downloaded Counts data was converted by $\log _{2}$ (FPKM+1). Quasi-Likelihood F-tests method of the package of EdgeR software [20] was used to analyze the expression difference of Cyclin D1, Cylin E1 and MMP9 between ccRCC and normal control tissues.

\section{Cell culture and stable cell construction}

The most highly cited and commonly used cell lines for ccRCC researches, including 786-O, ACHN and Caki-1 cells [2] were bought from Kunming institute of zoology, Chinese academy of sciences. Cell thawing was firstly conducted at $37^{\circ} \mathrm{C}$ water bath, centrifuged, removed cell freezing medium in ultra clean bench and transferred cells to the culture bottle. DMEM culture medium (1195500 bt, GIBCO, USA) containing 10\% FBS (10099141, GIBCO, USA) was added. Culture conditions were $37{ }^{\circ} \mathrm{C}, 5 \% \mathrm{CO}_{2}$ and saturated humidity. When cell convergence was about $80 \%$, the culture medium was abandoned, cells were washed with PBS for twice, and $1 \mathrm{~mL}$ of $0.25 \%$ of trypsin (25200072, GIBCO, USA) was added to digest cells for $1 \sim 2 \mathrm{~min}$. When most of the cell fell off, fresh culture medium containing serum was added and transferred to new culture bottles by 1:3.

Our previous results showed that Caki-1 cells had the highest G6PD activities in the three of RCC cell lines, which were much higher than that of the control HK2 cell lines. Whereas, 786-O and ACHN had the moderate and lowest activities of G6PD, respectively. Therefore, G6PD-overexpressing ACHN and 786-O or G6PD-knocked down Caki-1 and 786-O stable cells establishment were conducted as described before $[9,12,21]$. For G6PD-overexpressing ACHN cells construction, $2 \times 10^{5}$ ACHN cells were firstly seeded in a 6-well culture plate. When reaching $70-80 \%$ confluence, cells were transfected with $2 \mu \mathrm{g}$ pBABE-puro-G6PD or the control plasmid using Lipofectamine 2000. $48 \mathrm{~h}$ after transfection, $0.5 \mu \mathrm{g} / \mathrm{ml}$ puromycin was used for 3 weeks resistance selection. To construct G6PD-knocked down Caki-1 stable cells, $2 \times 10^{5}$ Caki-1 parental cells were firstly seeded in a 6-well culture plate. When reaching 70-80\% confluence, cells were transfected with $2 \mu \mathrm{g}$ pSR-GFP/Neo-G6PD shRNA or the control plasmid using Lipofectamine 2000. 48h after transfection, 1000 $\mu \mathrm{g} / \mathrm{ml} \mathrm{G418}$ was used for 3 weeks resistance selection.

\section{Real-time RT-PCR and Western blot analysis}

For real-time RT-PCR assay, total RNA was extracted from cells or tissues according to Trizol (15596-018, Invitrogen, USA) reagent instructions. cDNA was synthesized by reverse transcription according to Thermo RT Kit (K1622, Thermo, USA) instructions. Real-time PCR was performed using SYBR Green qPCR SuperMix (04913850001, Roche, Switzerland). Primers used were as follows: G6PD: F: 5'-TCATCATCATGGGTGCATCGG-3', R: 5'-CTT GAAGAAGGGCTCACTCTGTTTG-3'; Cyclin D1: F: 5'-GCGTACCCTGACACCCCTCTC-3', R: 5' -CTCCTC TTCGCCTGATCC-3'; Cyclin E1: F: 5' - ACTCAACGT GCAAGCCTCG-3', R: 5'- GCTCAAGAAAGTGCT GATCCC-3'; MMP9: F: 5'- AATCTCTTCTAGAGA CTGGGAAGGAG-3', R: 5'-AGCTGATTGACTAAA GTAGCTGGA-3'; U6: F: 5'-CTCGCTTCGGCA GCACA-3', R: 5'-AACGCTTCACGAATTTGCGT-3'.

For Western blot analysis, the total protein of cells or tissues was extracted with radioimmunoprecipitation assay (RIPA) protein lysis buffer (supplemented with PMSF) and quantified by bicinchoninic acid (BCA) method. SDS-PAGE with $10 \%$ separation gel and $5 \%$ compression gel were prepared. Equal amount of protein was loaded, and electrophoresis, membrane transfer and blocking with $5 \%$ non-fatty milk were performed. Afterward, the membranes were incubated with primary antibodies and then secondary antibody. The following antibodies were used: G6PD antibody (ab133525, Abcam, Cambridge, U.K.), Cyclin D1 antibody (ab16663, Abcam), Cyclin E1 antibody (ab33911, Abcam), MMP9 antibody (ab76003, Abcam), $\beta$-actin (\#4967, Cell Signaling Technology, Beverly, MA, USA), goat anti-rabbit IgG secondary antibody (sc2004, Santa Cruz Biotechnology) and goat 
anti-mouse IgG secondary antibody (sc2005, Santa Cruz Biotechnology). The results were detected by chemiluminescence method after washing the film with TBST solution. The results were analyzed by Image J grayscale scanning software.

\section{Cell cycle and proliferation assay}

For cell proliferation detection, $100 \mu \mathrm{L}$ cells suspension $\left(1 \times 10^{4} /\right.$ well $)$ were seeded into 96-well plates for $24 \mathrm{~h} .20 \mathrm{\mu l}$ of MTS reagent (CTB169, Promega, Beijing, China) was added to each well at different time points, and incubated for $2 \mathrm{~h}$ at $37^{\circ} \mathrm{C}$, followed by the measurement of absorbance at 490 $\mathrm{nm}$ using a microplate reader.

For cell cycle assay, cells were firstly seeded into 6-well plates and grown for $12 \mathrm{~h}$. After cultured in $0.2 \%$ FBS medium for $24 \mathrm{~h}$, cells were incubated in $10 \%$ FBS medium for another $24 \mathrm{~h}$. Cells were harvested and cell cycle analysis were performed as described in a previous report by a PARTEC CyFlow Space flow cytometer and ModFit software [12].

siRNA-Ctrl and siRNA-targeting Cyclin E1 (sequences: 5'-CACCCTCTTCTGCAGCCAA-3') were synthesized by Ribobio (Guangzhou, China). For siRNA transfection, $2 \times 10^{5} \mathrm{ACHN}$ cells were firstly seeded in a 6-well culture plate. When reaching $70-80 \%$ confluence, cells were transfected with $10 \mathrm{nM}$ siRNA-Ctrl or siRNA-targeting Cyclin E1 using Lipofectamine 2000. $48 \mathrm{~h}$ after transfection, cells were harvest and used for the following cell cycle, MTS and other experiments.

\section{Wound healing and Transwell assay}

For wound healing analysis, $1 \times 10^{5}$ cells were seeded into 6-well plates and cultured until $90 \%$ confluency. A $200 \mu \mathrm{L}$ pipette tip was used to make three parallel wounds in each well, and all wells were washed by PBS for twice. Cells were then cultured in serum-free medium and images were captured by inverted microscopy at 0 and $24 \mathrm{~h}$ after scratching. Cell migration distances were analyzed by using ImageJ software.

For Transwell migration analysis, $1 \times 10^{5}$ cells in $100 \mu \mathrm{l}$ of serum-free medium were seeded on the top surface of a 24-well ( $8 \mu \mathrm{M}$ Transwell membranes) and $600 \mu \mathrm{l}$ medium with 10\% FBS was added in the bottom of Transwell chambers. After incubation for 24 $\mathrm{h}$, the Transwell membranes were fixed with $4 \%$ formaldehyde for $20 \mathrm{~min}$ and stained with Crystal Violet for $10 \mathrm{~min}$ at room temperature. At last, cell numbers of 10 areas of each Transwell membrane, observed at $400 \times$ magnification, were analyzed and cell migration abilities of each cell line were assessed.

MMP9 specific inhibitor JNJ0966 was purchased from Selleck (S5696, Houston, TX, USA) and dissolved in $100 \%$ DMSO to prepare a $10 \mathrm{mM}$ stock and stored at $-20^{\circ} \mathrm{C}$. For cell treatment, the stock solution was added in the culture medium at a final concentration of $10 \mu \mathrm{M}$. Following $24 \mathrm{~h}$ stimulation, the migratory abilities of ccRCC cells were analyzed by Transwell assay as described above.

\section{Mice model}

A total of 20 six-week old BALB/c nude mice were purchased from the Beijing HFK Bioscience Co., Ltd (Beijing, China) and housed under pathogen-free conditions. All animal experiments were performed according to the guidelines of Animal Care and Use Committee of Kunming Medical University. A total of 20 mice were randomly divided into 4 groups and they were subcutaneously injected into their flanks with $1 \times 10^{6}$ ACHN- G6PDOE, Caki-1- G6PDsi, or relevant control cells, respectively. Tumor sizes were monitored every 5 days by using formula: (length $x$ width $\left.^{2}\right) \times 0.5$. The mice were euthanized after the last measurement and tumors were collected for further studies.

\section{MMP9 activity assay}

For MMP9 activity assay in stable transfected cells and xenograft mice model tissues, MMP9 assay kit for cell (GMS50088.1, Genmed, Shanghai, China) and MMP9 assay kit for tissue (GMS50088.2, Genmed, Shanghai, China) were used, respectively. This assay was dependent on the following principles: as matrix metalloproteinase substrate, gelatin was chemically modified by succinic anhydride to block its free amino group to react with 2,4,6-trinitrobenzenesulfonic acid; Once hydrolyzed by MMP9, major amines, including new reactive amino groups, are released and exposed and react with trinitrobenzene sulfonic acid to produce a chromatic reaction. Therefore, MMP9 activity was measured following the manufacturer's instruction and absorbances at OD $420 \mathrm{~nm}$ were detected by using spectrophotometer at $37^{\circ} \mathrm{C}$. The specific activity of MMP9 was calculated according to the gross activity subtract the nonspecific activity.

\section{Statistical analysis}

SPSS version 21.0 (IBM, Armonk, NY) was used for data statistical analysis. As the TCGA data was not normal distribution and the variance was uneven, the correlation between the expression of Cyclin D1, Cyclin E1, MMP9 and clinical parameters of ccRCC patients was analyzed by Mann-Whitney U test (two groups) or Kruskal-Wallis $\mathrm{H}(\mathrm{K})$ test (three groups). The survival analysis was investigated by Kaplan-Meier curves, and log-rank test was performed to measure the statistical difference. Genes high and low expression groups were made by employing the median cutoff values. Univariate and 
multivariate Cox regression models of survival were applied to analyze the prognostic values of genes expression and clinicopathologic features. The $\chi^{2}$ test was used for IHC analyses. Spearman correlation analysis was conducted by using TCGA data of both the normal and tumor tissues to evaluate the expression correlation between two different molecules. For other analysis, unpaired or paired Student's t-test was used. Error bars represent the means \pm standard deviation. $p<0.05$ indicates a significant statistical difference.

\section{Results}

\section{G6PD, Cyclin El and MMP9 are overexpressed in CCRCC and associated with poor outcomes in CCRCC patients}

To further unravel the underlying mechanisms of G6PD in ccRCC progression, 20 pairs of ccRCC tumor specimens and matched adjacent normal tissues were assessed by real-time RT-PCR, Western blot and IHC analysis. The results showed that the expression of G6PD, Cyclin E1 and MMP9 at both mRNA and protein expressions levels were elevated in human cCRCC tumors compared with adjacent normal tissues (Fig. 1A-I), indicating that highly expressed Cyclin E1 and MMP9 may be positively correlated with G6PD overexpression and synergistically involved in ccRCC tumorigenesis.

Previous studies from our research group demonstrate that G6PD could promote ccRCC cell proliferation and invasion through upregulating the expression of CyclinD1 and MMP2, respectively [9, 12]. Therefore, transcriptome sequencing data of 72 normal kidney tissues and 535 ccRCC cases were subsequently extracted from TCGA and subject to statistical analyses for further evaluating the expression profile and the role of the genes of interest including Cyclin D1, Cyclin E1, MMP2 and MMP9. The results of gene expression analyses showed that Cyclin D1, Cyclin E1 and MMP9 mRNA levels were significantly higher in ccRCC than that in normal tissues (Fig. 2A-C), whereas there was no significant difference between the expression level of MMP2 in cCRCC and normal control tissues (Supplement 1A). Moreover, MMP2 expression was not associated with cCRCC prognosis (Supplement 1B), indicating that G6PD mediated ccRCC progression may be depended on other more important underlying mechanisms. Subsequently, correlation analysis between the expression level of Cyclin D1, Cyclin E1, and MMP9 and clinicopathological features was performed. We observed a significant association between the expression levels of the three genes and the pathologic $\mathrm{T}$ stage, Fuhrman grade and TNM stage. However, only the expression levels of the proliferation-related genes Cyclin D1 and Cyclin E1 were significantly associated with the lymph node metastasis, and only the Cyclin E1 and MMP9 expression levels showed significant correlation with distant metastasis ( $M$ stage). Additionally, the expression levels of the three genes were significantly associated with the expression of G6PD in ccRCC specimens, indicating that all these genes may interact with G6PD in ccRCC tumorigenesis (Table 1).

To further examine the association between G6PD and these three genes, spearman correlation analysis was conducted using the TCGA data. As presented in Fig. 2D-F, the results showed that G6PD is positively correlated with Cyclin E1 $(r=0.455 ; p<$ $0.001)$ and MMP9 ( $r=0.385 ; p<0.001)$, but rather negatively correlated with Cyclin D1 $(r=-0.289 ; p<$ 0.001); suggesting that Cyclin E1 and MMP9 overexpression may be dependent on G6PD dysregulation in ccRCC. Taken together, these results indicate that these proliferation-and metastasisrelated factors, especially Cyclin E1 and MMP9, might be involved in G6PD mediated ccRCC progression, and correlated with ccRCC prognosis.

To evaluate the prognostic significance of the genes in ccRCC, all the $528 \mathrm{ccRCC}$ cases obtained from the TCGA were divided into high and low expression groups based on the median value of genes expression levels, Kaplan-Meier overall survival curves were plotted and log-rank test were conducted. The results demonstrated that patients with high Cyclin D1 expression level displayed a better prognosis (Fig. 2G). In addition, when the patients were separated into stage I/II $(n=320)$ and stage III/IV $(n=205)$ according to the TNM staging (3 patients with no specific staging in all the 528 cases), no significant association between Cyclin D1 expression and patients' survival was observed (Fig. 2H-I). Conversely, ccRCC patients with higher expression levels of Cyclin E1 and MMP9 had significantly shorter survival time than patients with low Cyclin E1 and MMP9 expression levels (Fig. 2J, M). Similarly, higher Cyclin E1 and MMP9 expression levels predicted worse survival rate in both ccRCC stage I/II and stage III/IV (Fig. 2K-L, N-O).

Furthermore, univariate Cox regression analysis revealed that high expression levels of G6PD, Cyclin E1 and MMP9, age at surgery, pathologic T stage, M stage, Fuhrman tumor grade, tumor laterality, as well as TNM stage were significant predictors of poor overall survival in ccRCC patients, whereas gender and $\mathrm{N}$ stage failed to be prognostic factors (Table 2). In addition, multivariate Cox regression analysis demonstrated that the expression of G6PD and Cyclin $\mathrm{E} 1$, as well as age at surgery, $\mathrm{M}$ stage and TNM stage 
were independent prognostic factors for ccRCC overall survival (Table 2). Taken together, these results indicate that G6PD, Cyclin E1 and MMP9 might play crucial role in the progression of ccRCC.
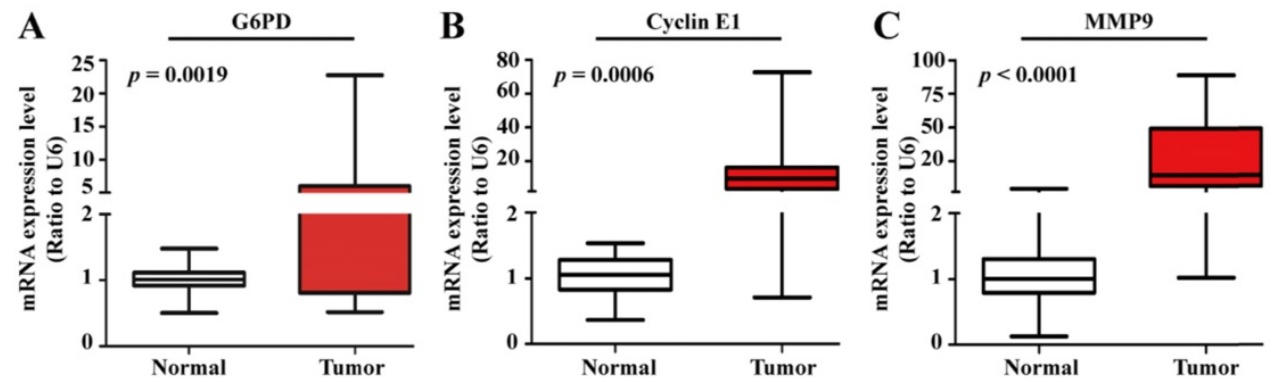

D
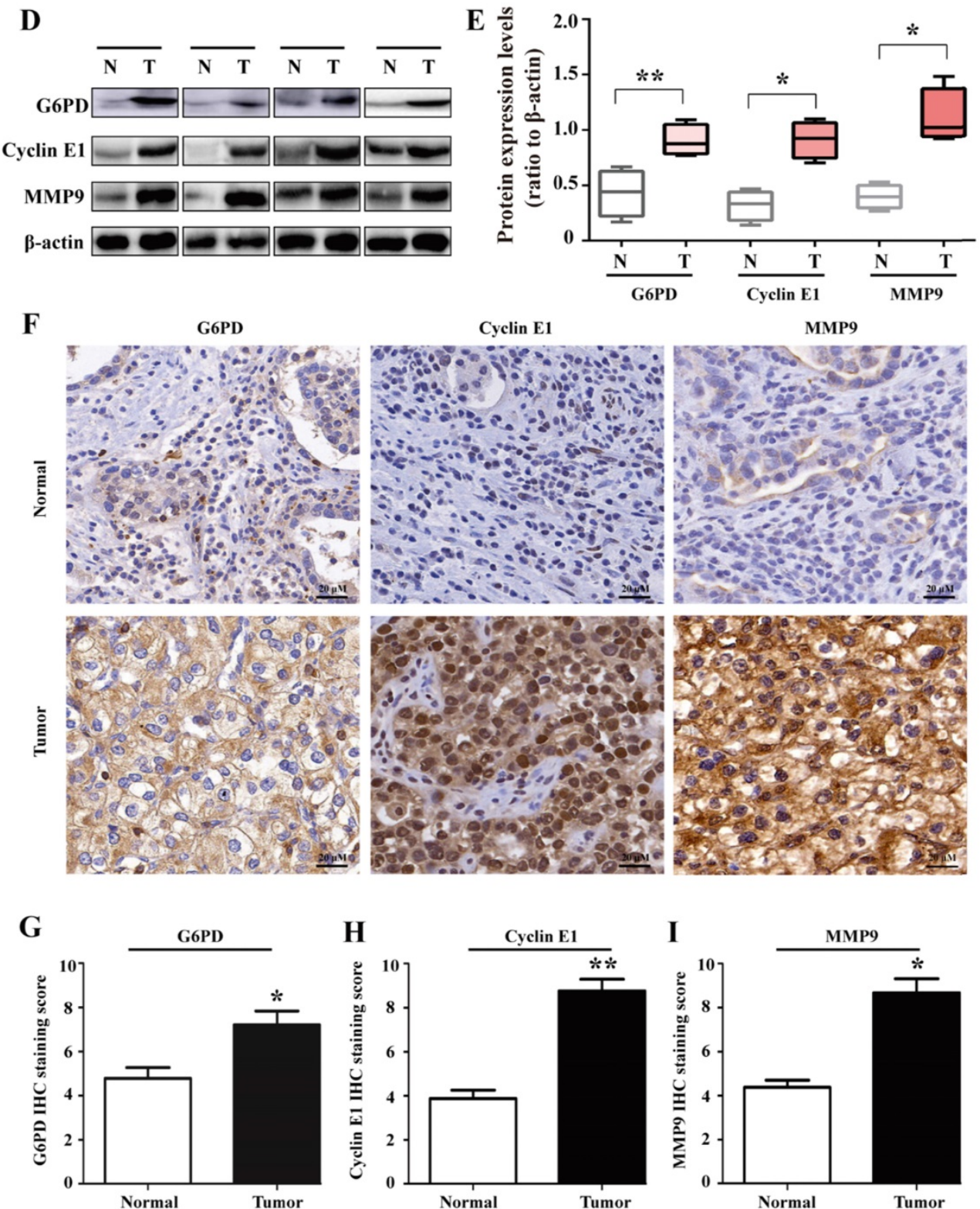

Figure 1. G6PD, Cyclin El and MMP9 are overexpressed in human ccRCC tissues. (A-E) real-time RT-PCR (A-C), Western blot (D) and grayscale scanning (E) were employed for the detection of G6PD, Cyclin E1 and MMP9 expression levels in ccRCC tumor specimens and relevant adjacent normal tissues ( $\mathrm{n}=20$ ). $\beta$-actin was used as a loading control. Representative cropped gels and blots of the Western blot analysis were shown (D). The samples used for quantitative comparisons in the Western blot analysis were derived from the same experiment and that gels were processed in parallel (E). (F-I) IHC were conducted to analyze the expression of G6PD, Cyclin E1 and MMP9 in ccRCC and relevant adjacent normal tissues $(n=20)$. Representative images were shown $(\mathbf{F})$. Statistical analysis was conducted by paired Student's $t$-test for Western blot analysis $(\mathbf{E})$ and by $X^{2}$ test for IHC analysis $(\mathbf{G}-\mathbf{I})$, respectively. $* p<0.05, * * p<0.01$ vs. Normal tissues. 

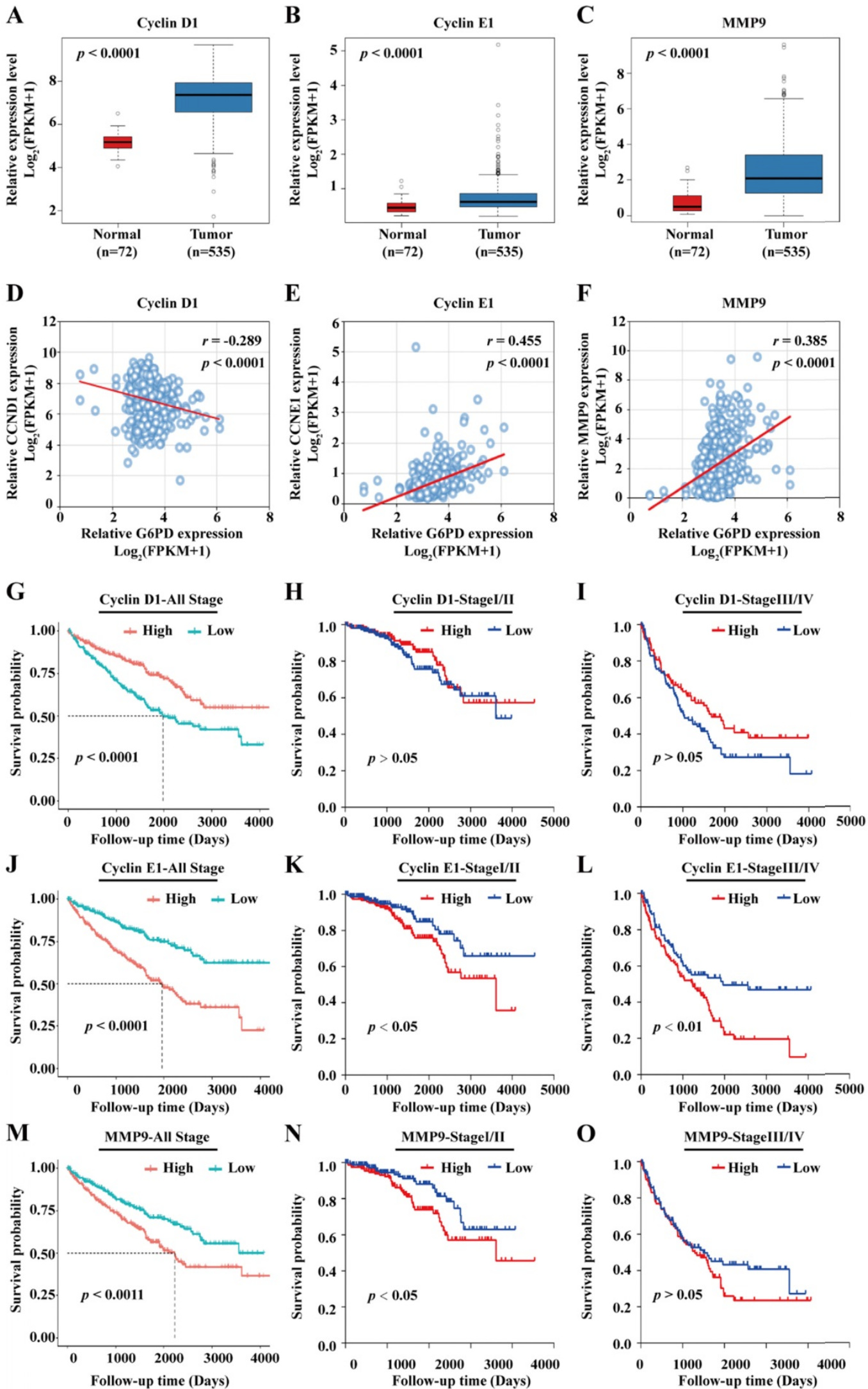

Figure 2. Cyclin El and MMP9 are positively correlated with G6PD and associated with poor outcomes in ccRCC patients. (A-C) mRNA expression levels of Cyclin DI, Cyclin EI and MMP9 in normal kidney tissues $(n=72)$ and ccRCC specimens $(n=535)$ were analyzed by TCGA dataset mining (Mann-Whitney U test). (D-F) Spearman correlation analyses between G6PD and Cyclin DI, G6PD and Cyclin EI, G6PD and MMP9 at the mRNA expression levels were conducted in ccRCC and normal kidney tissues. (G-O) Kaplan-Meier analyses for overall survival of all ccRCC patients $(n=528)$, patients with stage l/II ccRCC $(n=320)$ and patients with stage III/IV ccRCC ( $n=205)$ in the TCGA cohort with high vs. low indicated gene mRNA expression levels were shown (log-rank test). 
Table 1. Correlations between the expression of Cyclin D1, Cyclin E1, MMP9 and important clinicopathological variables in ccRCC.

\begin{tabular}{|c|c|c|c|c|c|c|c|}
\hline \multirow[t]{2}{*}{ Parameters } & \multirow[t]{2}{*}{ Case No. } & \multicolumn{2}{|l|}{ Cyclin D1 } & \multicolumn{2}{|l|}{ Cyclin E1 } & \multicolumn{2}{|l|}{ MMP9 } \\
\hline & & Expression & $P$ value & Expression & $P$ value & Expression & $P$ value \\
\hline \multicolumn{8}{|l|}{ Sex } \\
\hline Male & 344 & 7.040 & $<0.001^{\mathrm{a}}$ & 0.732 & $0.109^{a}$ & 2.559 & $0.227^{a}$ \\
\hline Female & 184 & 7.428 & & 0.710 & & 2.436 & \\
\hline \multicolumn{8}{|l|}{ Age } \\
\hline$<60$ & 245 & 7.124 & $0.194^{\mathrm{a}}$ & 0.717 & $0.439 a$ & 2.417 & $0.316^{\mathrm{a}}$ \\
\hline$\geq 60$ & 283 & 7.219 & & 0.732 & & 2.601 & \\
\hline \multicolumn{8}{|l|}{ T stage } \\
\hline $\mathrm{T} 1 / 2$ & 340 & 7.326 & $<0.001^{\mathrm{a}}$ & 0.663 & $<0.001^{\mathrm{a}}$ & 2.215 & $<0.001^{a}$ \\
\hline $\mathrm{T} 3 / 4$ & 188 & 6.902 & & 0.836 & & 3.060 & \\
\hline \multicolumn{8}{|l|}{ N stage } \\
\hline No & 239 & 7.145 & $0.003^{b}$ & 0.701 & $<0.001^{b}$ & 2.597 & $0.096^{b}$ \\
\hline N1 & 16 & 6.364 & & 1.257 & & 3.210 & \\
\hline $\mathrm{Nx}$ & 273 & 7.248 & & 0.714 & & 2.405 & \\
\hline \multicolumn{8}{|l|}{ M stage } \\
\hline M0 & 420 & 7.269 & $0.064^{\mathrm{b}}$ & 0.678 & $<0.001^{b}$ & 2.498 & $<0.001^{b}$ \\
\hline M1 & 78 & 6.681 & & 0.857 & & 2.930 & \\
\hline $\mathrm{Mx}$ & 28 & 7.174 & & 0.768 & & 1.539 & \\
\hline NA. & 2 & 6.602 & & 0.655 & & 3.783 & \\
\hline \multicolumn{8}{|l|}{ Laterality } \\
\hline Right & 279 & 7.244 & $0.583^{\mathrm{b}}$ & 0.694 & $0.050^{\mathrm{b}}$ & 2.493 & $0.469^{b}$ \\
\hline Left & 248 & 7.097 & & 0.759 & & 2.547 & \\
\hline Bilateral & 1 & 7.193 & & 0.912 & & 1.140 & \\
\hline \multicolumn{8}{|c|}{ Fuhrman grade } \\
\hline G1/2 & 240 & 7.433 & $<0.001^{\mathrm{b}}$ & 0.617 & $<0.001^{b}$ & 2.177 & $<0.001^{b}$ \\
\hline G3/4 & 280 & 6.984 & & 0.801 & & 2.824 & \\
\hline Gx & 5 & 5.661 & & 0.839 & & 1.634 & \\
\hline NA. & 3 & 6.909 & & 2.060 & & 2.326 & \\
\hline \multicolumn{8}{|l|}{ TNM stage } \\
\hline $\mathrm{I} / \mathrm{II}$ & 320 & 7.358 & $<0.001^{\mathrm{b}}$ & 0.658 & $<0.001^{b}$ & 2.200 & $<0.001^{b}$ \\
\hline III/IV & 205 & 6.895 & & 0.827 & & 2.992 & \\
\hline Discrepancy & 3 & 6.778 & & 0.895 & & 3.663 & \\
\hline \multicolumn{8}{|c|}{ G6PD expression } \\
\hline Low & 264 & 6.341 & $<0.001^{\mathrm{a}}$ & 0.458 & $<0.001^{a}$ & 1.253 & $<0.001^{a}$ \\
\hline High & 264 & 8.009 & & 0.991 & & 3.779 & \\
\hline
\end{tabular}

Abbreviations: NA., Not Available. a, Mann-Whitney U test; b, Kruskal-Wallis H (K) test. Significant $p$-value was in bold.

Table 2. Univariate and multivariate Cox regression analyses of the association of G6PD, Cyclin D1, Cyclin El and MMP9 expression and other clinicopathologic features with overall survival in ccRCC.

\begin{tabular}{|c|c|c|c|c|c|c|}
\hline \multirow[t]{2}{*}{ Characteristics } & \multicolumn{3}{|c|}{ Univariate } & \multicolumn{3}{|c|}{ Multivariate } \\
\hline & HR & $95 \% \mathrm{CI}$ & $p$ value & HR & $95 \% \mathrm{CI}$ & $p$ value \\
\hline Sex & 0.952 & $0.698-1.297$ & 0.753 & & & \\
\hline Age & 1.786 & $1.304-2.447$ & $<0.001$ & 1.638 & $1.190-2.255$ & 0.002 \\
\hline T stage & 3.021 & $2.232-4.089$ & $<0.001$ & 0.704 & $0.402-1.236$ & 0.222 \\
\hline N stage & 0.914 & $0.786-1.063$ & 0.243 & & & \\
\hline M stage & 2.131 & $1.693-2.681$ & $<0.001$ & 1.493 & $1.135-1.965$ & 0.004 \\
\hline Laterality & 1.393 & $1.034-1.877$ & 0.029 & 1.339 & $0.993-1.806$ & 0.056 \\
\hline Fuhrman grade & 2.142 & $1.602-2.864$ & $<0.001$ & 1.379 & $0.980-1.942$ & 0.065 \\
\hline TNM stage & 3.699 & $2.730-5.012$ & $<0.001$ & 2.583 & $1.853-3.601$ & $<0.001$ \\
\hline G6PD expression & 1.959 & $1.547-2.482$ & $<0.001$ & 1.423 & $1.070-1.892$ & 0.015 \\
\hline Cyclin D1 expression & 0.770 & $0.682-0.871$ & $<0.001$ & 0.975 & $0.834-1.140$ & 0.751 \\
\hline Cyclin E1 expression & 1.713 & $1.422-2.062$ & $<0.001$ & 1.447 & $1.113-1.881$ & 0.006 \\
\hline MMP9 expression & 1.193 & $1.099-1.295$ & $<0.001$ & 1.030 & $0.935-1.135$ & 0.546 \\
\hline
\end{tabular}

Abbreviations: HR, hazard ratio; CI, confidence interval. Significant $p$-value was in bold.

\section{G6PD upregulates the expression of Cyclin E1 and MMP9 in vitro}

To confirm that the interplay between G6PD and aforementioned genes is necessary for ccRCC progression, we first evaluate the related genes expressions in ACHN-G6PDOE, Caki-1-G6PD ${ }^{\text {si }}$ and control cells by real-time RT-PCR and Western blot respectively. The results demonstrated that G1/S transition- and proliferation-related gene Cyclin E1 was significantly increased by approximately 1.1-fold at the mRNA level plus 1.5-fold at the protein level in ACHN-G6PD ${ }^{\mathrm{OE}}$ cells, whereas the expression levels of Cyclin E1was reduced by about $54.1 \%$ at the mRNA level plus $51.2 \%$, respectively at the protein level in Caki-1-G6PD ${ }^{\text {si }}$ (Fig. 3A-C).

Regarding the cell migration-related gene, our results showed that MMP9, the matrix metalloproteinase which exhibited the largest fold change between ccRCC and normal control tissues [17], had not been changed significantly at the mRNA level when G6PD was overexpressed or knocked 
down. In contrast, the Western blot results showed that MMP9 was significantly increased by about 2-fold in ACHN-G6PDOE, whereas it was decreased by $45.6 \%$ in Caki-1-G6PD ${ }^{\text {si }}$ (Fig. 3A-C). Furthermore, the enzyme activity analysis also demonstrated that when G6PD was overexpressed, a 0.9-fold increase of MMP9 activity was detected in ACHN-G6PDOE cells compared with the control, whereas G6PDknockdown resulted in an approximately $45.0 \%$ of MMP9 activity reduction in Caki-1-G6PDsi cells compared with control cells (Fig. 3D). Overall, these results suggest that G6PD-mediated ccRCC progression probably require the upregulation of Cyclin E1 and MMP9.

\section{G6PD changes cell cycle dynamics and facilitates CCRCC cells growth}

Given that G6PD and Cyclin E1 are overexpressed and positively correlated, we aimed to elucidate their possible interplay in ccRCC cells proliferation. To do so, the cell cycle profiles were analyzed in ACHN- G6PDOE, Caki-1- G6PD si and relevant control cells. As presented in Fig. 4A-B, in ACHN- G6PDOE cells, the cell population of G0/G1 phase was significantly decreased by approximate $34.3 \%$, while the cell population of $S$ and G2/M phases showed an obvious increase compared to that of the control. In contrast, G6PD-knockdown (Caki-1G6PD ${ }^{\text {si) }}$ resulted in a 0.3-fold increase in the G0/G1 fraction and a decrease in the $S$ and G2/M phase compared to that of the control (Non-silencer) (Fig. 4C-D). These results indicated that G6PD might promote ccRCC cells proliferation through promoting the G1/S transition and changing the cell cycle distribution. The results of subsequent MTS assay confirmed that overexpression of G6PD in ACHN cells significantly increased the cell growth rate by about 3.6-fold at day 5 after seeding compared to that of the control cells (Fig. 4E). Meanwhile, when G6PD was knocked down, an approximate $27.8 \%$ decreased proliferation rate was observed in Caki-1-G6PD ${ }^{\mathrm{Si}}$ cells at day 5 compared to that of the Non-silencer cells (Fig. 4F). Taken together, these results suggest that G6PD might facilitate ccRCC cells proliferation through the regulation of cell cycle progression by modulating Cyclin E1 expression.
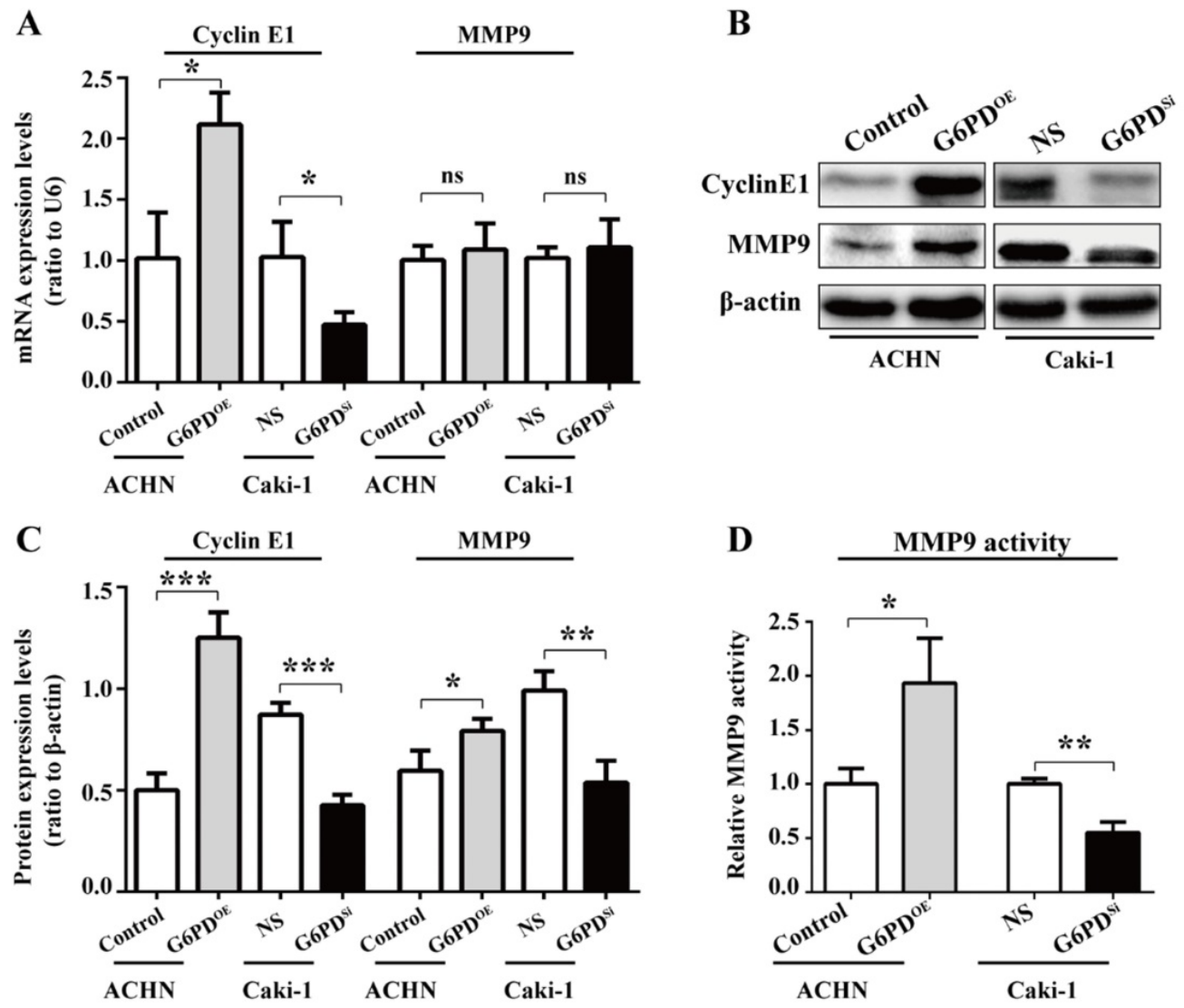

Figure 3. G6PD upregulates the expression of Cyclin E1 and MMP9 in vitro. (A-C) The expression of Cyclin E1 and MMP9 at the mRNA and protein level in stably transfected ACHN-G6PDOE, Caki-1- G6PDi and relevant control cells was analyzed by using real-time RT-PCR (A), Western blot and grayscale scanning assay (B-C), respectively. $\beta$-actin was used as a loading control. Representative cropped gels and blots of the Western blot analysis were shown (B). The samples used for quantitative comparisons in the Western blot analysis were derived from the same experiment and that gels were processed in parallel (C). (D) Relative MMP9 enzyme activities in ACHN-G6PDOE, Caki-1- G6PDsi and relevant control cells were analyzed by using MMP9 activity kit in stable transfected ACHN or Caki-1 cells. All assays were done in at least triplicate. Bars represent the means \pm SD. $*_{p}<0.05,{ }^{*} p<0.01$, ***p $<0.001$ vs. Control or Non-silencer (unpaired Student's $t$-test). 


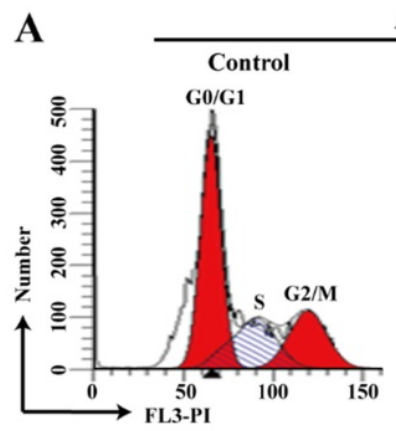

ACHN

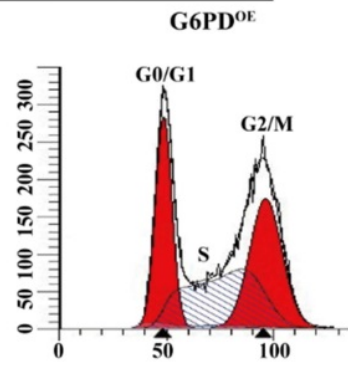

B

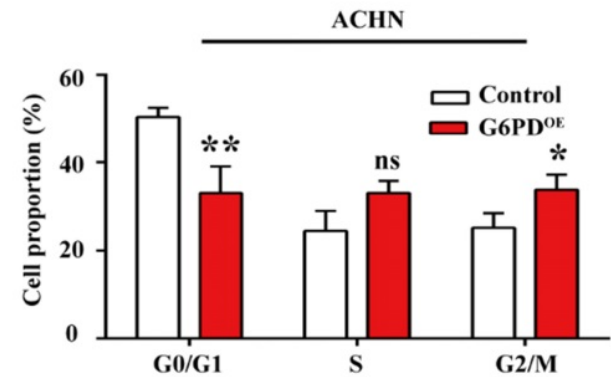

E

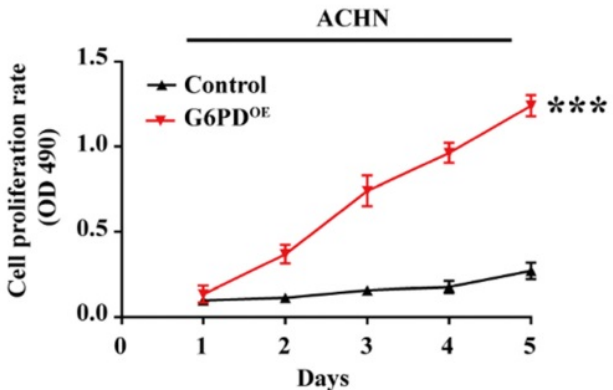

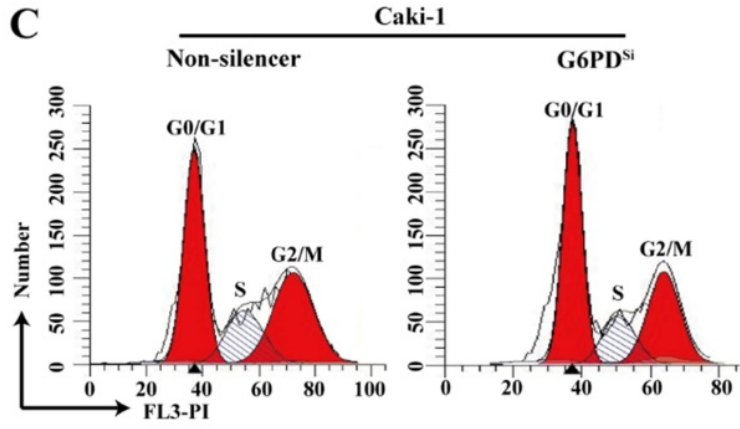

D

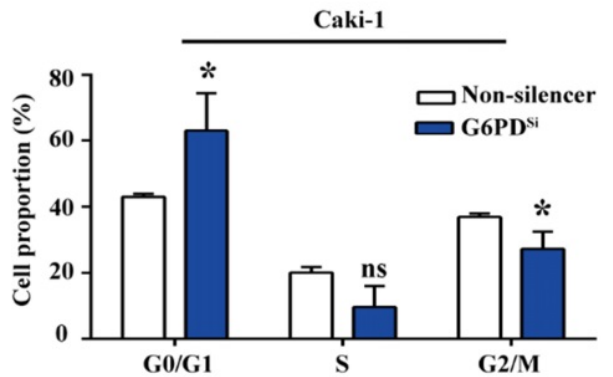

F

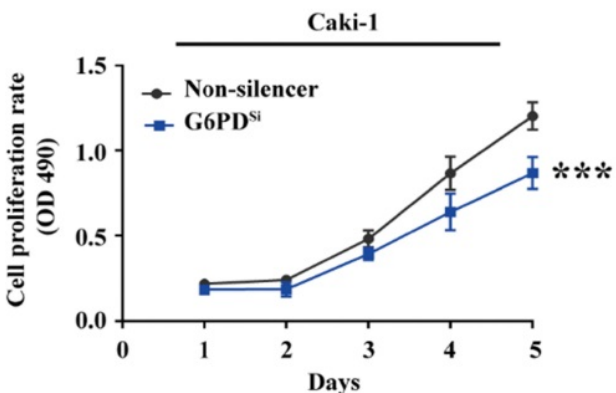

Figure 4. G6PD changes cell cycle dynamics and facilitates ccRCC cells growth. (A-D) Stably transfected ACHN-G6PDOE, Caki-1- G6PDsi and relevant control cells were subjected to cell cycle distribution analysis by PI staining and flow cytometry assay. (E-F) Cell proliferation abilities of ACHN-G6PDOE, Caki-1- G6PDsi and relevant control cell lines were assessed by MTS assay at different time points ( $1 \sim 5$ day). The statistical data represented three independent experiments, each performed in triplicate. Error bars represent the means \pm SD. ns, Not significant, ${ }^{*} p<0.05$, ${ }^{*} p<<0.01$, *** $p<0.001$ vs. Control or Non-silencer group (unpaired Student's $t$-test for B-C, Mixed ANOVA for E-F).

\section{G6PD enhances the migration ability of ccRCC cells}

Our previous study revealed that G6PD could promote ccRCC invasion through mediating MMP2 [12]. However, how G6PD mediates the progression of ccRCC to metastasis is still not clear. As increased cell migration was an important aspect in metastasis and positively correlated with the degree of malignancy and the mortality of ccRCC patients, wound healing assay and transwell analysis were performed using 786-O/ACHN-G6PDOE, 786-O/ Caki-1-G6PD ${ }^{\mathrm{Si}}$ and relevant control cells to evaluate whether G6PD imparted the migration ability of ccRCC cells. The results showed that wound healing ability was increased by $30.4 \%$ in $786-\mathrm{O}-\mathrm{G} 6 \mathrm{PD}$ OE cells at 24 hours (Fig. 5A-B), while decreased about 26.2\% in 786-O-G6PD ${ }^{\mathrm{Si}}$ cells compared with the Non-silencer cells (Fig. 5C-D). Moreover, the transwell analysis demonstrated that G6PD overexpression could increase the migration ability of $\mathrm{ACHN}-\mathrm{G} 6 \mathrm{PD}^{\mathrm{OE}}$ cells by about 3.0-fold compared to that of the control (Fig. 5E-F). In contrast, about $68.6 \%$ decreased cell mobility was observed in the Caki-1-G6PD ${ }^{\mathrm{Si}}$ cells compared to that in the Non-silencer cells (Fig. 5G-H). The above evidences indicate that G6PD could promote the migration ability of ccRCC cells.

\section{Cyclin E1 and MMP9 are involved in the G6PD-mediated cCRCC cells proliferation and migration}

All the above results imply that G6PD-mediates proliferation and migration may be heavily dependent on the up-regulation of Cyclin E1 and MMP9 in ccRCC cells. For further clarify this notion, a series of experiments were conducted to clarify whether the Cyclin E1 and MMP9 levels could affect the G6PD overexpression facilitated ccRCC cells proliferation and migration, respectively. Caki- 1 cells with relative high expression of G6PD, Cyclin E1 and MMP9 were chosen for Cyclin E1 knockdown and MMP9 activity inhibition, and subsequently for genes 
function detection. Whereas, ACHN-Control cells with relative low gene expression levels were used for the revers experiment along with the ACHN-G6PDOE cells. Firstly, the Cyclin E1 siRNA transfection efficiency in Caki-1, ACHN-Control and ACHN-G6PDOE cells was testified by real-time RT-PCR and Western blot respectively. The results demonstrated that Cyclin E1 expression levels were significantly decreased at the mRNA and protein level in both Caki-1 and ACHN-G6PD ${ }^{\mathrm{OE}}$ cells (Fig. 6A-B). Subsequently, the cell cycle dynamics and cell proliferation rates were analyzed by using flow cytometry and MTS assay, respectively.

As presented in Fig. 6C-D, in Caki-1 cells, the
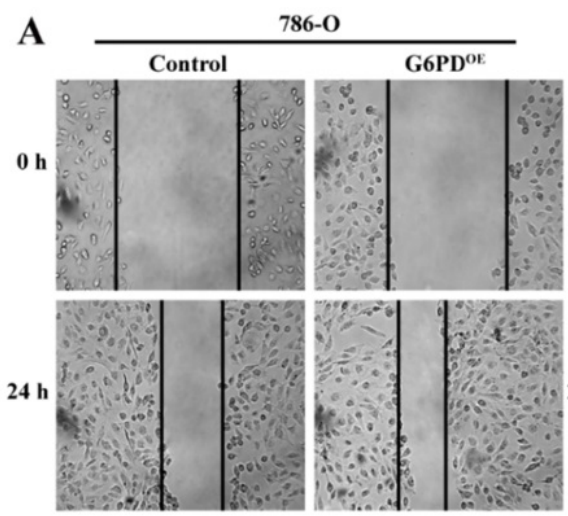

B

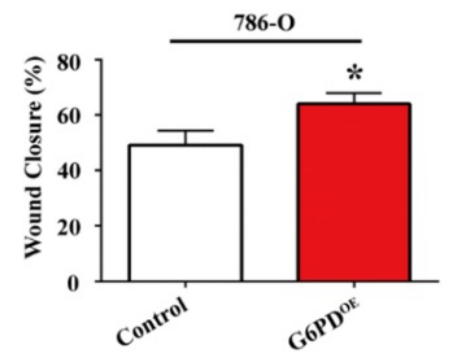

$\mathbf{E}$

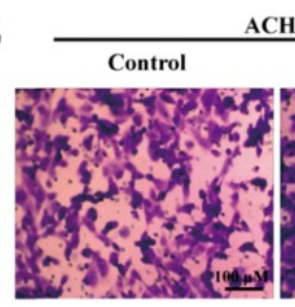

$\mathbf{F}$

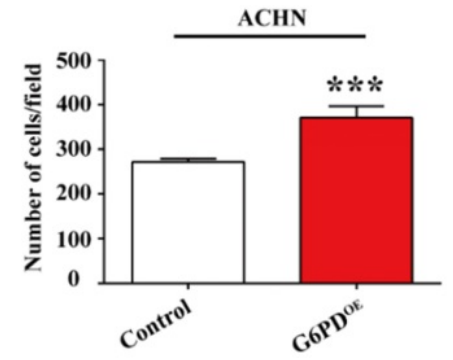

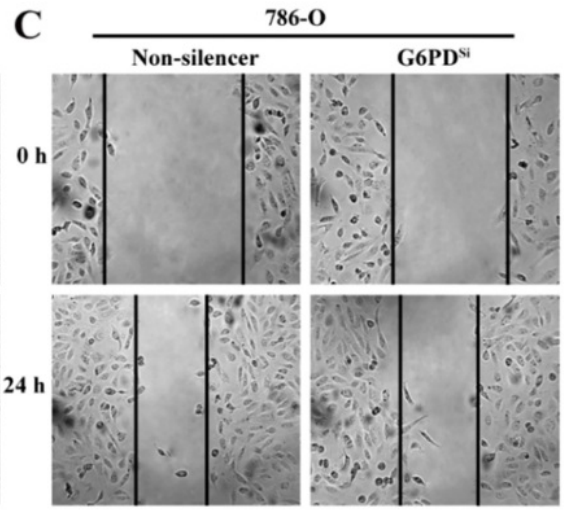

cell population of G0/G1 phase was significantly increased by approximate $11.1 \%$ following the Cyclin E1 siRNA transfection, while the cell population of $S$ and G2/M phases showed an obvious decrease compared to that of the control. Meanwhile, as what we confirmed previously in Fig. 4A-B, G6PD-overexpression (ACHN-G6PDOE) could result in an obvious decrease in the G0/G1 fraction and an increase in the $S$ and G2/M phase compared to that of the control. Whereas, Cyclin E1 siRNA reversed the G6PD -overexpression promoted cell cycle progression and induced cell cycle arrest in G0/G1 phase (Fig. 6C-D).

D

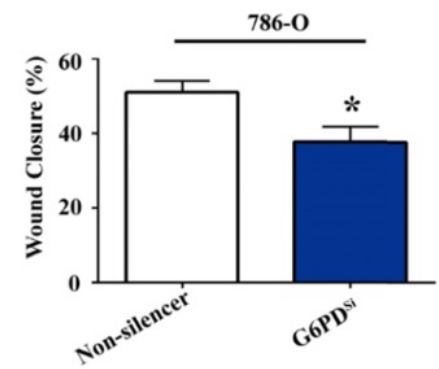

G
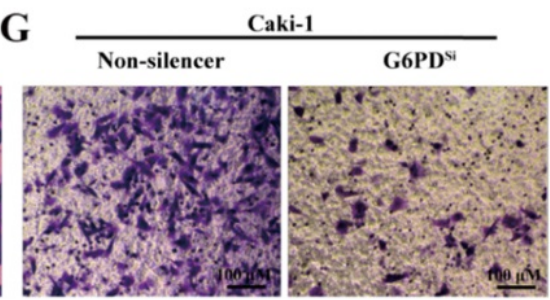

H

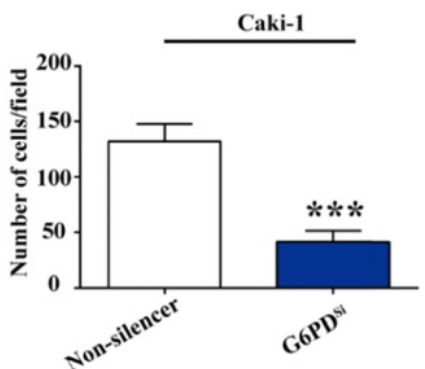

Figure 5. G6PD enhances the migration ability of ccRCC cells. (A) Wound-healing assay was performed to determine the effect of G6PD on migration abilities of 786-O-G6PDOE, 786-O- G6PDsi and relevant control cells. (B) ACHN-G6PDOE, Caki-1- G6PDsi and relevant control cells were subjected to Transwell assays. Representative images $(\mathbf{A}, \mathbf{C}, \mathbf{E}, \mathbf{G})$ and quantification analyses $(\mathbf{B}, \mathbf{D}, \mathbf{F}, \mathbf{H})$ are shown. The statistical data represented three independent experiments, each performed in triplicate. Error bars represent the means \pm SD. ${ }^{*} p<0.05,{ }^{*} p<0.01,{ }^{* *} p<0.001$ vs. Control or Non-silencer group (unpaired Student's $t$-test). 
A

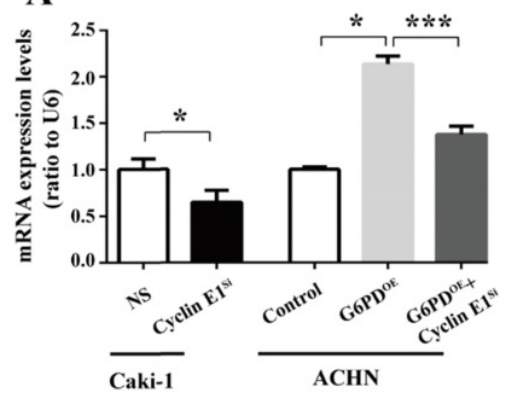

C

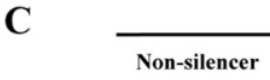

Caki-1

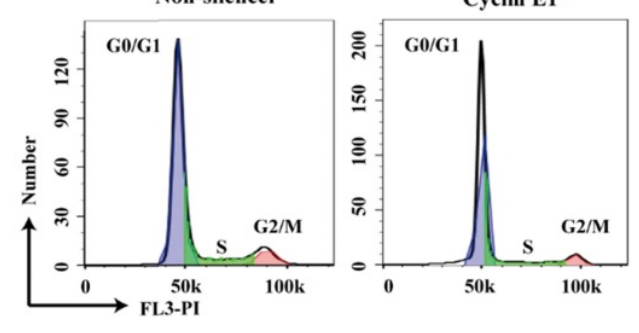

D

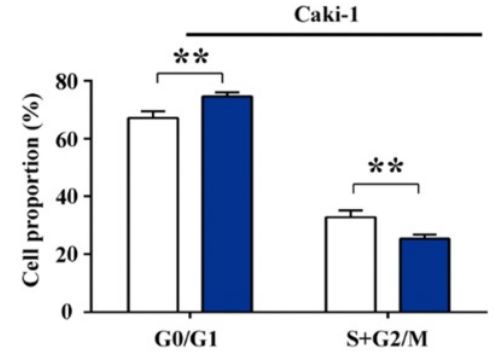

G

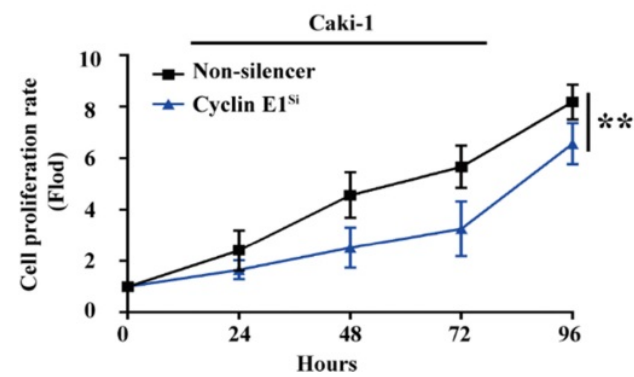

E
B

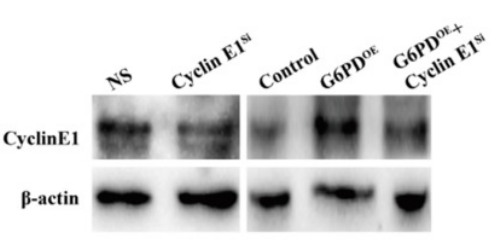

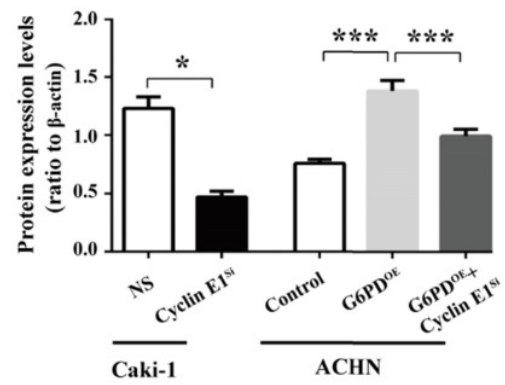
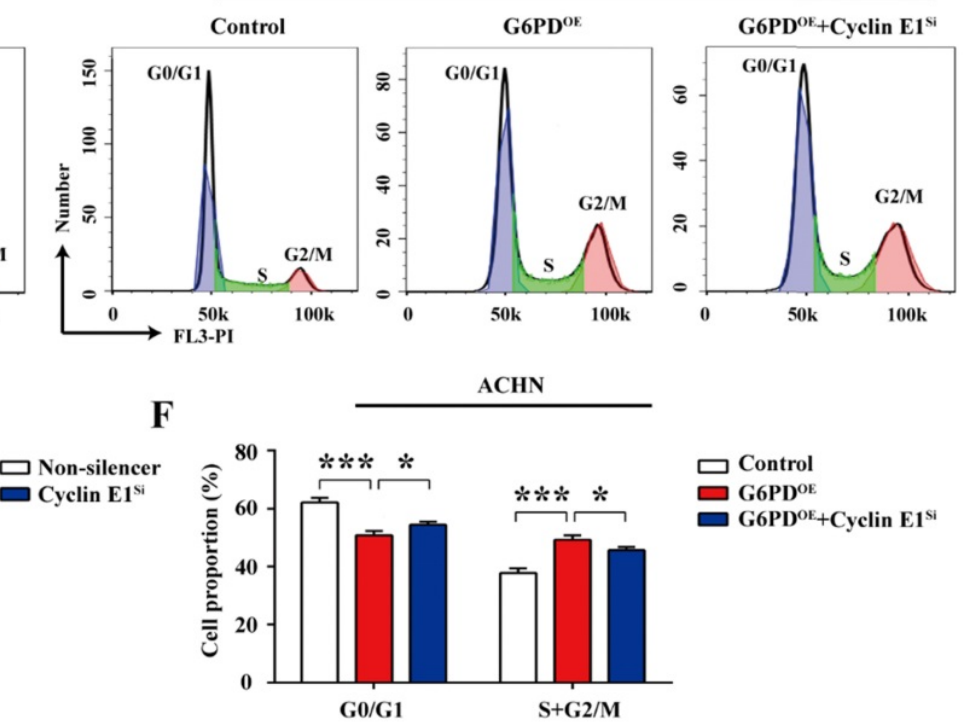

H

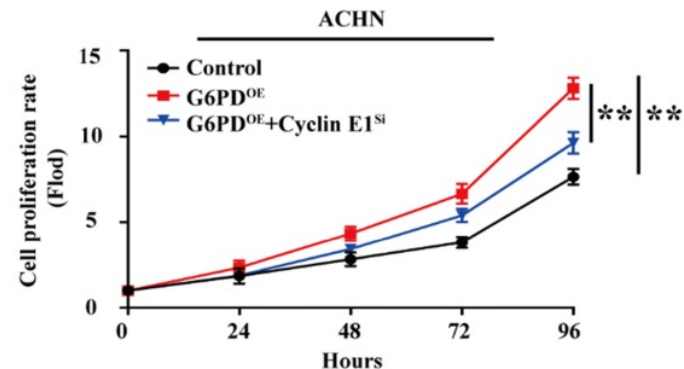

I

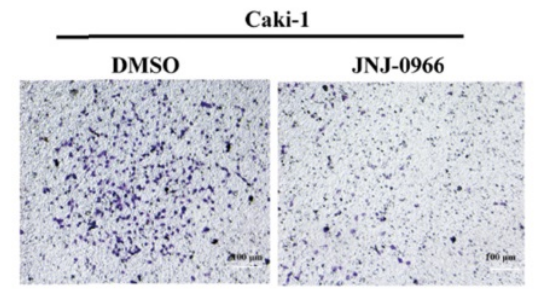

K

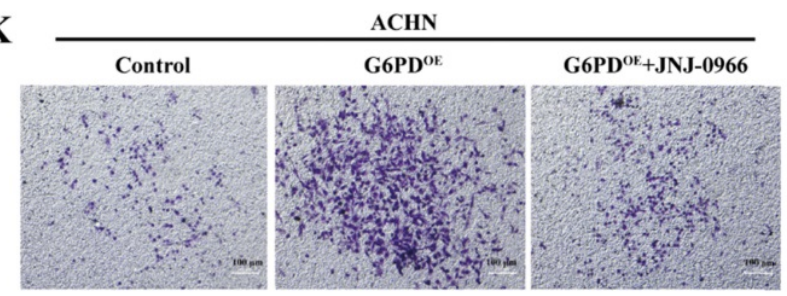

$\mathbf{J}$

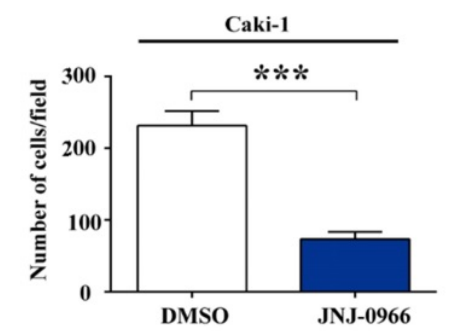

M

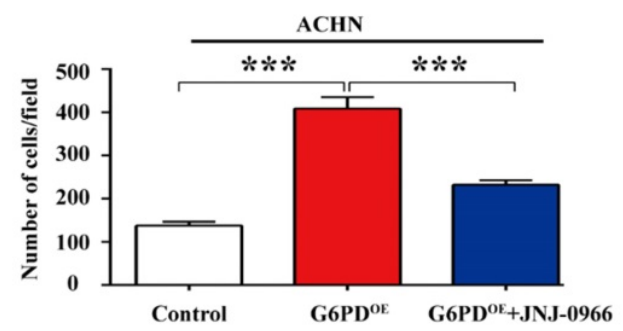

Figure 6. Cyclin E1 and MMP9 are involved in the G6PD-mediated ccRCC cells proliferation and migration. (A-B) The expression of Cyclin El at the mRNA and protein level in Caki-1, ACHN-G6PDOE and relevant control cells was analyzed by using real-time RT-PCR (A) and Western blot assay (B), respectively at 48 h after Cyclin E1 siRNA transfection. $\beta$-actin was used as a protein loading control. Representative cropped gels and blots of the Western blot analysis were shown (B). (C-F) Cyclin El siRNA transfected Caki-1, ACHN-G6PDOE and relevant control cells were subjected to cell cycle distribution analysis by PI staining and flow cytometry assay. (G-H) Cell proliferation 
abilities of Caki-1-Cyclin Elsi, ACHN-G6PDOE-Cyclin El si and relevant control cell lines were assessed by MTS assay at different time points. (I-M) Caki-1, ACHN-G6PDOE cells following treatment with the MMP9 inhibitor JNJ-0966 (10 $\mu \mathrm{M}, 24 \mathrm{~h})$ and relevant control cells were subjected to Transwell assays. Representative images (I, K) and quantification analyses (J, M) are shown. All assays were done in at least triplicate. Bars represent the means \pm SD. $* p<0.05$, $* * p<0.01$, $* * * p<0.001$ vs. relevant control (Mixed ANOVA for G-H, unpaired Student's $t$-test for others)
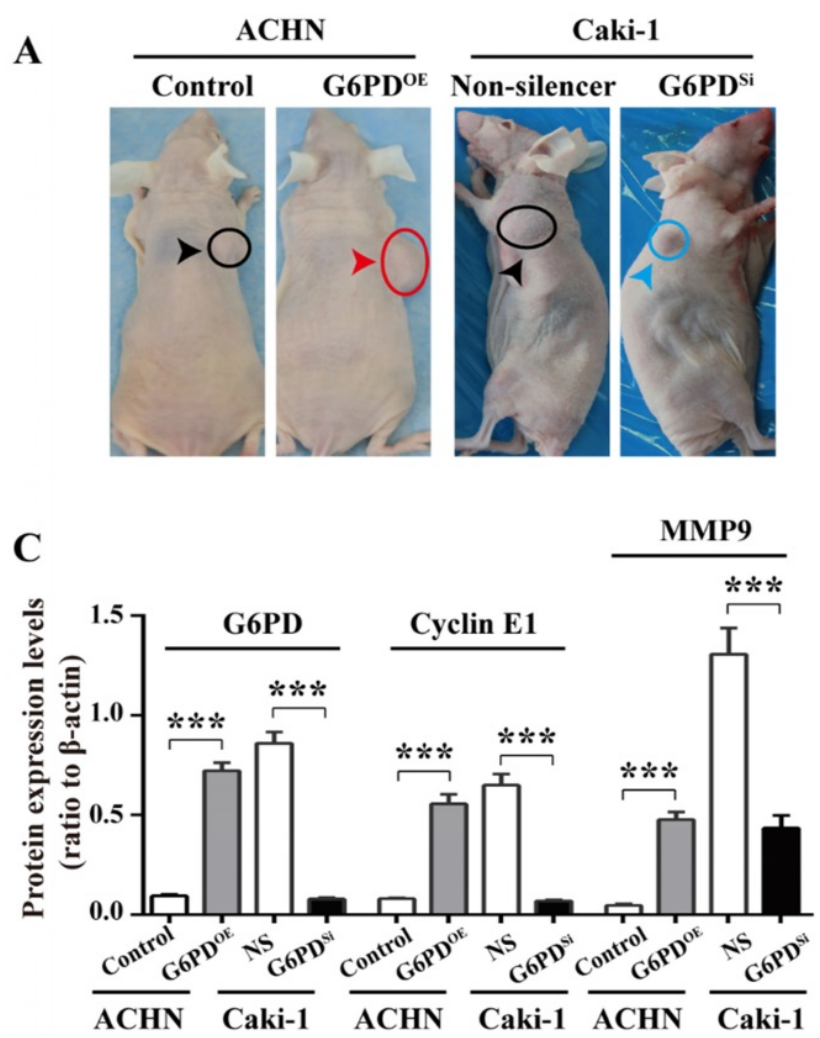
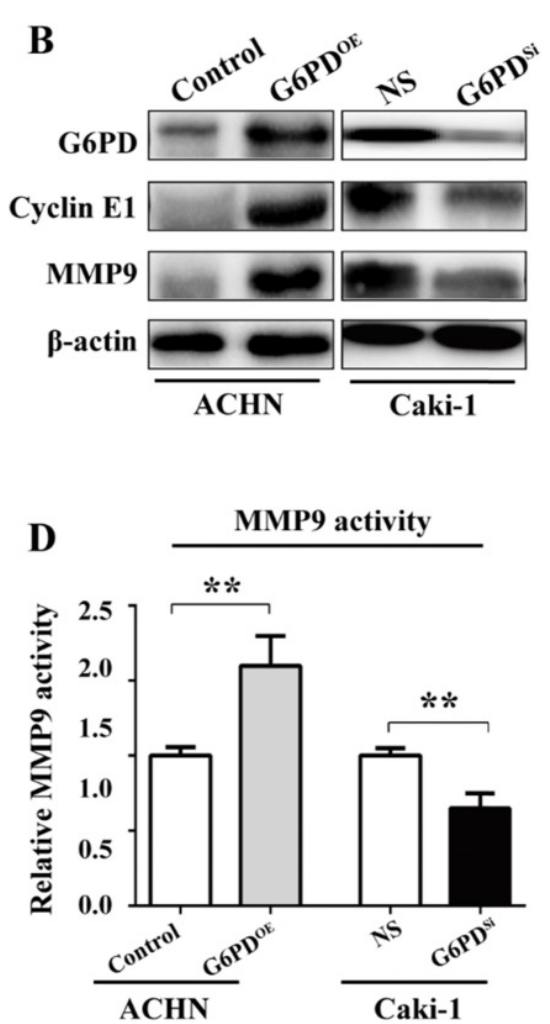

Figure 7. G6PD upregulates Cyclin E1 and MMP9 to enhance ccRCC progression in vivo. (A) Stably transfected ACHN-G6PDOE, Caki-1- G6PDsi and relevant control cells were subcutaneous injected in the nude mice, respectively. Representative xenografted mice images were shown. (B-C) The protein expression of G6PD, Cyclin E1 and MMP9 in the mice tumor tissue were analyzed by Western blot analysis (B) and grayscale scanning (C). $\beta$-actin served as a loading control. Representative cropped gels and blots of the Western blot analysis were shown (B). The samples used for quantitative comparisons in the Western blot analysis were derived from the same experiment and that gels were processed in parallel (C). (D) Relative MMP9 enzyme activities in the mice tumor tissue were analyzed by using MMP9 activity kit. The data represent three independent experiments. Each bar represented the mean \pm SD. $*^{*} p<0.01$, $* * * p<0.001$ vs. Control or Non-silencer (unpaired Student's $t$-test).

The results of subsequent MTS assay showed that when Cyclin E1 was knocked down, an approximate $19.8 \%$ decreased proliferation rate was observed in Caki-1 cells at 96 hours after seeding compared to that of the Non-silencer cells (Fig. 6G). Meanwhile, Fig. $\mathbf{6 H}$ confirmed that overexpression of G6PD in ACHN cells significantly increased the cell growth rate by about 0.6 -fold at 96 hours after seeding compared to that of the control cells, but this cell proliferation promoting effect of G6PDoverexpression could be obviously reversed by approximate $24.7 \%$ following Cyclin E1 knockdown. The above evidences indicate that Cyclin E1 might be an important regulatory target gene of G6PD mediated signaling pathways in the proliferation of cCRCC.

Furthermore, JNJ0966, a specific therapeutic inhibitor of MMP-9, which is commonly used for many researches, including cancer, fibrosis, immune dysregulation, and neurodegenerative diseases, could inhibit activation of MMP-9 zymogen and subsequent generation of catalytically active enzyme, but have no effect on MMP-1, MMP-2, MMP-3, MMP-9, or MMP-14 catalytic activity and does not inhibit activation of the highly related MMP-2 zymogen. Therefore, JNJ0966, the highly selective compound, was used to treat ccRCC cells at the concentration of $10 \mu \mathrm{M}$ for $24 \mathrm{~h}$ in our current study for MMP9 activity inhibition $[22,23]$. The results of subsequent transwell analysis demonstrated that about $68.3 \%$ decreased cell mobility was observed in the Caki-1 cells following treatment with the MMP9 inhibitor JNJ-0966 (Fig. 6I-J), while the upregulated migration ability could be reversed by about $43.2 \%$ following JNJ-0966 stimulation in G6PD-overexpressing ACHN cells (Fig. 6K-M). These results suggest that MMP9 is required for the G6PD enhanced migration ability of ccRCC cells. Taken together, these evidences suggest that Cyclin E1 siRNA and MMP9 inhibitor could reverse G6PD upregulated ccRCC cells proliferation and migration, which indicating that these functional genes including Cyclin E1 and MMP9 may probably be necessary regulatory factors and involved in the G6PD-mediated ccRCC proliferation and migration. 


\section{G6PD upregulates Cyclin E1 and MMP9 expression in the xenografted cCRCC mice model}

To further back up the importance of G6PD-mediated Cyclin E1 and MMP9 overexpression in the progression of ccRCC, in vivo study was conducted as described in one of our previous reports [12]. Xenografted nude mice models were constructed by subcutaneously injecting ACHN-G6PDOE, Caki-1-G6PD ${ }^{\mathrm{Si}}$ or relevant control cells. The results revealed that $\mathrm{ACHN}-\mathrm{G} 6 \mathrm{PD} \mathrm{OE}^{\mathrm{E}}$ cells produced larger tumor compared with control, whereas Caki-1-G6PDsi parental cells induced smaller tumors compared with the Non-silencers (Fig. 7A). Subsequently, the mice tumor tissues were subjected to Western blot analysis. As presented in Fig. 7B-C, the expression levels of G6PD, Cyclin E1 and MMP9 protein were significantly increased in $\mathrm{ACHN}-\mathrm{G} 6 \mathrm{PD}^{\mathrm{OE}}$-derived tumor tissues, whereas they were obviously decreased in Caki-1-G6PDSi-derived tumor tissues compared with the corresponding controls. Moreover, about 0.6-fold increased MMP9 activity was detected in ACHN-G6PD ${ }^{\mathrm{OE}}$-derived tumor tissues compared with the control, whereas the MMP9 activity was decreased by about 35\% in Caki-1-G6PDSi-derived tumor tissues (Fig. 7D). Taken together, these results demonstrate that G6PD upregulates Cyclin E1 and MMP9 expression in the xenografted ccRCC mice model, which indicate that G6PD may promote ccRCC progression through facilitating the expression of both Cyclin E1 and MMP9.

\section{Discussion}

It has been reported that about $33 \%$ of RCC has already metastasized at the first diagnosis, and $20 \%$ $50 \%$ of patients will progress to metastasis following surgery $[1,6]$. Despite ccRCC treatment has developed for decades, the advanced and metastatic ccRCCs are still challenging due to its resistance to chemo- and radiotherapy, therefore RCC patients are still confronted with worse prognosis [1]. Although enormous efforts about identifying appropriate biomarkers for ccRCC tumorigenesis, progression and aggressiveness have been made to improve the efficiency of ccRCC diagnosis and prognosis, to date fewer particular biomarkers exhibit satisfactory potential for ccRCC classification and prognosis prediction or is ready for widespread use in clinical application $[1,24]$. Therefore, one of the main aims of present study is to investigate the underlying mechanism of ccRCC progression and identify new biomarkers that are associated with ccRCC tumor development and clinical parameters, which may be helpful for ccRCC earlier diagnosis and prognosis, and may even become novel therapeutic options and improve the survival of ccRCC patients.

The present study scrutinizes the hypothesis that the biological function and mechanism of G6PD-mediated ccRCC progression involve the modulation of Cyclin E1 and MMP9 expressions. Moreover, the results suggested that the interplay between G6PD, Cyclin E1 and MMP9 is more likely to be implicated in the development of ccRCC rather than Cyclin D1 and MMP2. G6PD was shown, in our previous study, to promote ccRCC proliferation by upregulating the Cyclin D1 expression. However, recent studies delineate controversy about the prognostic role of Cyclin D1 in RCC [25]. Some study indicated that low expression of Cyclin D1 was linked to large tumor size, high nuclear grade, and poor prognosis of ccRCC patients [15]. A very recent meta-analysis of 18 studies with 2282 RCC patients demonstrated that high Cyclin D1 expression level was positively associated with better prognosis of RCC patients in disease free survival rate, but there was no association between overall survival and Cyclin D1 expression in ccRCC patients [25]. Our present and previous studies supported that Cyclin D1 expression is high in ccRCC and modulated by G6PD [9]. However, both genes were not positively correlated, and ccRCC patients with high Cyclin D1 expression showed better prognosis. Additionally, some studies have even suggested non-oncogenic role for Cyclin D1, and down-regulated Cyclin D1 could increase the cell invasion and improve the outcome of breast cancer patients [25]. These findings indicated that Cyclin D1 performed roles besides oncogenic and might exert functions in impairing the malignant potential of ccRCC.

As an important cell cycle regulator, the classic function of Cyclin D1 is to form a complex with cyclin dependent kinase (CDK) 4/6 and promote G1/S transition [25]. However, the catalytic partners of Cyclin D1, CDK 4 and CDK6, did not provide satisfactory results either. We found that there was no significant difference between the expression level of CDK4 presented in ccRCC and normal tissues, whilst CDK6 expression was conversely decreased in ccRCC specimens compared with the control. Moreover, neither CDK4 nor CDK6 showed any prognostic significance on the impact of ccRCC patients' survival (data not shown). These aforementioned controversies prompt us to identify other more accurate proliferation-related factor that could be regulated by G6PD and involved in ccRCC tumorigenesis. The present study demonstrated that the G1/S transition regulator Cyclin E1 was highly expression in ccRCC and could be a potential biomarker for ccRCC prognosis. This finding is strongly consistent with previous reports that 
demonstrated the oncogenic function of Cyclin $\mathrm{El}$ in cancers. For instance, its oncogenic role was highlighted in breast cancer [26], ovarian cancer [27] and osteosarcoma [28]. More interestingly, Cyclin E1 could be mediated by G6PD overexpression and high Cyclin E1 expression predicted poor outcomes, which indicated that as a cell cycle-related molecular, Cyclin E1 might be a more crucial downstream target of G6PD in promoting ccRCC tumor proliferation.

Previous study from our laboratory demonstrated that G6PD is overexpressed in ccRCC and has the ability to promote tumor cell proliferation and invasion $[9,12]$. However, whether G6PD could enhance ccRCC migration and the underlying regulatory mechanisms are remains unknown. MMPs are intriguing genes related to cancer progression, and they have been found to exert crucial regulatory roles in cell apoptosis, migration, angiogenesis and immunity. Increasing evidences demonstrate that MMPs are commonly upregulated in types of human cancers and associated with patient prognosis. For instance, MMP1, 3, 9 and 10-14 were highly expressed in breast cancer, colon adenocarcinoma, esophageal cancer, head and neck cancer, etc. However, some MMPs are downregulated in some cancers, such as MMP2 and 23B in breast cancer, bladder cancer, lung squamous cancer and uterine corpus endometrial carcinoma [17]. The expression of representative MMPs were also measured in ACHN-G6PDOE, Caki-1 $\mathrm{G} \mathrm{PD}^{\mathrm{Si}}$ and relevant control cells in our study [12]. The previous and present results showed that MMP2 and MMP9 had the most significant protein expression changes when G6PD was overexpressed or knocked down in ccRCC.

MMP2 and MMP9, also known as gelatinase A and gelatinase $\mathrm{B}$, are considered to be the major MMPs involved in invasion and metastasis of numbers cancers because of their capacity to degrade the important components of basement membranes, including laminin, gelatin, nidogen, type I and IV collagens [19]. Nevertheless, as Cyclin D1, the prognostic role of MMP2 in RCC is controversial. Some reports showed that MMP2 is overexpressed in RCC, involved in RCC invasion and angiogenesis, and correlated with poor outcome of RCC patients $[12,29]$. However, our present study, together with other reports, found no significant difference of MMP2 expression in the analysis of large numbers of ccRCC clinical samples and normal kidney tissue [17]. Furthermore, we found that MMP2 expression level is not associated with ccRCC prognosis (Supplement 1). Otherwise, MMP9 has been found to have the largest fold change between ccRCC and normal control tissues [17]. Consistent with this, our study also found that MMP9 was highly expressed in ccRCC tissues, and it predicted poor outcomes in ccRCC patients. Of note, the current study demonstrated that MMP9 expression can be regulated by G6PD in ccRCC. In fact, both genes are positively correlated in ccRCC. However, the multivariate Cox regression analysis showed that the expression MMP9 was not included in the independent prognostic factors for ccRCC survival, which suggested the complications of identifying potential biomarkers for ccRCC [24]. Though the current findings do not yet provide an immediate clinical application, some essential clues are revealed and more researches are required in future investigation.

Our current study demonstrated that both MMP9 protein expression level and activity could be upregulated by G6PD through the ccRCC cell model and xenografted mice model analyses. Intriguingly, we observed that the mRNA expression of MMP9 was not obviously changed following G6PD overexpression or knockdown in ccRCC cell lines, which seemed to be inconsistent with the results of TCGA dataset mining which indicated both genes were highly expressed and positively correlated at the mRNA level in ccRCC tumor specimens and normal control.

The possible reasons for these inconsistences were as follows. Firstly, the TGGA dataset was dependent on the transcriptome sequencing, whereas, our mRNA detection in G6PD overexpressing or knocked down ccRCC cells was conducted by real-time RT-PCR analysis. Both of these two different methods and currently available other technologies are perfectly accurate in neither mRNA or protein quantifications. These factors potentially influence the detection of biologically truly significant correlations [30]. Secondly, the TCGA transcriptional expression analyses of G6PD and MMP9 were test results of human ccRCC tumor specimens and normal control kidney tissues, which were different from the detection of mRNA and protein expression levels based on stably transfected cell models. Therefore, different biological or experimental mRNA and protein degradation rates might affect the mRNA and protein correlations [30]. Thirdly, gene expression could be regulated at several levels including pre-transcriptional, transcriptional and posttranscriptional levels, and any variation in regulatory link, zymogen activatiocn, enzyme concentration and hence activity may result in enhanced MMP9 function of promoting tumor migration and progression in ccRCC. Although some gene mRNA was detected, there may be no indication that the mRNA was translated into protein [31], and vice versa, in some cases, high expression levels of protein may lead to stress response which results in decreased mRNA 
transcription [31, 32]. Therefore, some mRNA samples allow good estimations about the corresponding protein expression, but for some others, pronounced deviation could be observed [33, 34].

Protein and functional activity of a gene showed a significant correlation in most cases, but there could be no correlation between any of these parameters and mRNA levels, such as the cytochrome P4502E1, a constitutively expressed gene in human liver $[32,34]$. In our present study, G6PD upregulated protein expression and functional activity of MMP9 demonstrated that G6PD could facilitate ccRCC progression partially through promoting MMP9 function. However, the inconsistent expression of MMP9 gene at the mRNA and protein levels, when G6PD was overexpressed or knocked down in ccRCC cell lines, might be depended on the stress caused by exogenous gene transfection. When level of one protein was too high, it may cause some kind of stress on cell. To maintain balance within cells and save energy, cells inevitably reduced the gene transcription, whereas, cells may promote transcription, when low level of protein was translated [30, 32, 34]. Compared with the different testing object from TCGA transcriptome sequencing, stable transfected ccRCC cell lines were stressed by G6PD OE/KD and the following significant modification of MMP9 protein and activity, which may rather result in little change of MMP9 at the mRNA levels.

Taken together, the results of TCGA dataset mining indicated that G6PD, Cyclin E1 and MMP9 were overexpressed and positively correlated in ccRCC, which were just hints, but not causation. The present study scrutinizes the hypothesis that the biological function and mechanism of G6PDmediated ccRCC progression involve the modulation of Cyclin E1 and MMP9 expressions. The little changes of MMP9 at the mRNA level in ccRCC cell models does not affect our core conclusion of this report. However, it is an interesting phenomenon and meaningful work that needs further study.

Thus, our present study, supported with strong methodology, provide novel therapeutic pathway, involving G6PD, Cyclin E1 and MMP9, that can be considered in future for ccRCC treatment. It is well established that G6PD is critical in the maintenance of the redox equilibrium in the cell. It preserves the cell homeostasis by regulating ROS production and elimination [11]. Doing so, G6PD sustains the high level of ROS in cancer cells while instigating their survival. In fact, it has been reported that ROS dysregulation is an important factor leading to abnormal signal transduction in cells $[35,36]$. ROS promotes and interacts with numerous oncogenic signaling pathways, such as the STAT3, MAPK and
NF-kB pathways, to favor the development of human cancers. Moreover, proliferation and metastasis related genes, such as cyclins and MMPs, which were transcriptional regulation targets of these oncogenic signaling transducers [37-40] and were found to be involved in this ROS-mediated mechanisms of action in ccRCC $[12,21,41]$. These implies that ROS and its relevant signaling pathways might be involved in the G6PD-mediated upregulation of Cyclin E1 and MMP9 in our study. However, to elucidate the exact regulatory mechanism, some more in vitro and in vivo experiments should be conducted in the future investigation.

Intriguingly, we observed that the mRNA expression of MMP9 was not significantly modified when G6PD was overexpressed or knocked down. We hypothesized that it may be resulting from some epigenetic modifications. The absence of mRNAprotein correlation for a subset of investigated genes suggested that the relation between mRNA and protein was not strictly linear, but had a more intrinsic and complex dependence, deviating from the classical view referred to as the molecular dogma. Different regulation mechanisms, such as synthesis and degradation rates, acting on both the synthesized mRNA and the synthesized protein, affected the amount of the two molecules differentially [30, 32, 34]. In the current study, G6PD probably increased MMP9 protein stability through some intermediate mediator and other regulatory mechanisms. Additionally, it has been proved that ROS can activate MMPs or regulate MMP9 mRNA stability and lead to the destruction of extracellular matrix and facilitate tumor metastasis $[19,42]$, which promote us to hypothesize that facilitated MMP9 activation in ccRCC may also be induced by the G6PD-mediated ROS accumulation. Moreover, it has been well confirmed that G6PD was involved in certain carcinogenesis, and served key roles in extensive cancer cell metabolic reprogramming, including affection the amino acid metabolic pool [43-45], which may in turn provide building blocks for MMP9 protein synthesis. Hence, how G6PD, a cytoplasmic enzyme, regulates Cyclin E1 and MMP9 overexpression, and which signaling pathway serves as a mediator between these aberrations are unknown and required to be clarified in future studies.

\section{Conclusion}

In summary, the present study corroborates the oncogenic role of G6PD in ccRCC and extends the involved molecular mechanisms. The results indicated that G6PD changed cell cycle dynamics, facilitated cell proliferation, promoted migration in vitro, and enhanced ccRCC tumor growth in vivo, 
probably by upregulating Cyclin E1 and MMP9. Moreover, G6PD was positively correlated with Cyclin E1 and MMP9, all being highly expressed in human ccRCC tissues and associated with poor ccRCC prognosis. These findings reveal the feasibility of G6PD, Cyclin E1 and MMP9 as novel biomarkers and pave ways for the development of novel therapeutics for ccRCC.

\section{Supplementary Material}

Supplementary figure.

https://www.medsci.org/v19p0047s1.pdf

\section{Acknowledgments}

This work was supported by the National Natural Science Foundation of China (No. 81960462, $31960145,81760455,31960200,81560037,81460421$, $31660246,81660135,81760426,82103388,82160581$ and 82160540) and the Yunnan Province Applied Basic Research Funds (No. 2018FE468(-001), 2018FB120 and 2019FB091).

\section{Statement to all experimental protocols}

All human participants involved experiments were approved by the Ethics Committee of Kunming Medical University, according to the regulations of the Declaration of Helsinki. All animal experiments were approved by the Institutional Animal Care and Use Committee, Kunming medical University, according to the regulations for the Administration of Affairs Concerning Experimental Animals (China, 1988). Other experimental protocols were approved by the Department of Biochemistry and Molecular Biology, School of Basic Medical Sciences, Kunming Medical University.

\section{Statement to all methods}

All methods were carried out in accordance with relevant guidelines and regulations as described in the Materials and Methods section of this study.

\section{Consent to participate} consent.

All patients provided prior written informed

\section{Availability of data and material}

The data used to support the findings of this study are available from the corresponding author upon request.

\section{Author Contributions}

$\mathrm{YZ}$ and $\mathrm{ZY}$ were responsible for the experiments design and results interpretation. $\mathrm{YK}$ and $\mathrm{ZiY}$ collected the human ccRCC specimens. YN, XY and ZiY performed the IHC analysis. SW, WL, BS and QS conducted the TCGA assay. QH performed the cell cycle and MTS assay with the help of $\mathrm{HB}, \mathrm{YuZ}$ and LY. YN and QZ performed the other experiments. YZ and QZ organized figures and wrote the manuscript with the help of YA and ZY. All authors approved the final version of this manuscript.

\section{Competing Interests}

The authors have declared that no competing interest exists.

\section{References}

1. Jones TM, Carew JS, Nawrocki ST. Therapeutic Targeting of Autophagy for Renal Cell Carcinoma Therapy. Cancers. 2020; 12.

2. Sinha $R$, Winer AG, Chevinsky $M$, Jakubowski $C$, Chen $Y B$, Dong $Y$, et al. Analysis of renal cancer cell lines from two major resources enables genomics-guided cell line selection. Nature communications. 2017; 8: 15165.

3. Global Burden of Disease Cancer C, Fitzmaurice C, Abate D, Abbasi N, Abbastabar H, Abd-Allah F, et al. Global, Regional, and National Cancer Incidence, Mortality, Years of Life Lost, Years Lived With Disability, and Disability-Adjusted Life-Years for 29 Cancer Groups, 1990 to 2017: A Systematic Analysis for the Global Burden of Disease Study. JAMA oncology. 2019.

4. Hsieh JJ, Le V, Cao D, Cheng EH, Creighton CJ. Genomic classifications of renal cell carcinoma: a critical step towards the future application of personalized kidney cancer care with pan-omics precision. The Journal of pathology. 2018; 244: 525-37.

5. Hakimi AA, Reznik E, Lee CH, Creighton CJ, Brannon AR, Luna A, et al. An Integrated Metabolic Atlas of Clear Cell Renal Cell Carcinoma. Cancer cell. 2016; 29: 104-16.

6. Dannenmann SR, Hermanns T, Bransi A, Matter C, von Boehmer L, Stevanovic $\mathrm{S}$, et al. Spontaneous peripheral T-cell responses toward the tumor-associated antigen cyclin D1 in patients with clear cell renal cell carcinoma. Cancer immunology research. 2013; 1: 288-95.

7. Nagashio R, Oikawa S, Yanagita K, Hagiuda D, Kuchitsu Y, Igawa S, et al. Prognostic significance of G6PD expression and localization in lung adenocarcinoma. Biochimica et biophysica acta Proteins and proteomics. 2019; 1867: 38-46.

8. $\mathrm{Pu} \mathrm{H}$, Zhang $\mathrm{O}$, Zhao C, Shi L, Wang $\mathrm{Y}$, Wang J, et al Overexpression of G6PD is associated with high risks of recurrent metastasis and poor progression-free survival in primary breast carcinoma. World journal of surgical oncology. 2015; 13: 323.

9. Zhang Q, Yi X, Yang Z, Han Q, Di X, Chen F, et al. Overexpression of G6PD Represents a Potential Prognostic Factor in Clear Cell Renal Cell Carcinoma. Journal of Cancer. 2017; 8: 665-73.

10. Yang HC, Wu YH, Yen WC, Liu HY, Hwang TL, Stern A, et al. The Redox Role of G6PD in Cell Growth, Cell Death, and Cancer. Cells. 2019; 8.

11. Zhang C, Zhang Z, Zhu Y, Qin S. Glucose-6-phosphate dehydrogenase: a biomarker and potential therapeutic target for cancer. Anti-cancer agents in medicinal chemistry. 2014; $14: 280-9$

12. Zhang Q, Han Q, Yang Z, Ni Y, Agbana YL, Bai H, et al. G6PD facilitates clear cell renal cell carcinoma invasion by enhancing MMP2 expression through ROS-MAPK axis pathway. International journal of oncology. 2020 : 57: 197-212.

13. Gayed BA, Youssef RF, Bagrodia A, Kapur P, Darwish OM, Krabbe LM, et al. Prognostic role of cell cycle and proliferative biomarkers in patients with clear cell renal cell carcinoma. The Journal of urology. 2013; 190: 1662-7.

14. Hedberg Y, Davoodi E, Ljungberg B, Roos G, Landberg G. Cyclin E and p27 protein content in human renal cell carcinoma: clinical outcome and associations with cyclin D. International journal of cancer. 2002; 102: 601-7.

15. Hedberg Y, Ljungberg B, Roos G, Landberg G. Expression of cyclin D1, D3, E, and p27 in human renal cell carcinoma analysed by tissue microarray. British journal of cancer. 2003; 88: 1417-23.

16. Gonzalez-Avila G, Sommer B, Mendoza-Posada DA, Ramos C, Garcia-Hernandez AA, Falfan-Valencia R. Matrix metalloproteinases participation in the metastatic process and their diagnostic and therapeutic applications in cancer. Critical reviews in oncology/hematology. 2019; 137: 57-83.

17. Gobin E, Bagwell K, Wagner J, Mysona D, Sandirasegarane S, Smith N, et al. A pan-cancer perspective of matrix metalloproteases (MMP) gene expression profile and their diagnostic/prognostic potential. BMC cancer. 2019; 19: 581.

18. Slaton JW, Inoue K, Perrotte P, El-Naggar AK, Swanson DA, Fidler IJ, et al. Expression levels of genes that regulate metastasis and angiogenesis correlate with advanced pathological stage of renal cell carcinoma. The American journal of pathology. 2001; 158: 735-43.

19. Burlaka AP, Ganusevich, II, Gafurov MR, Lukin SM, Sidorik EP. Stomach Cancer: Interconnection between the Redox State, Activity of MMP-2, MMP-9 and Stage of Tumor Growth. Cancer microenvironment : official journal of the International Cancer Microenvironment Society. 2016; 9: 27-32. 
20. Lun AT, Chen Y, Smyth GK. It's DE-licious: A Recipe for Differential Expression Analyses of RNA-seq Experiments Using Quasi-Likelihood Methods in edgeR. Methods in molecular biology. 2016; 1418: 391-416.

21. Zhang Q, Yang Z, Ni Y, Bai H, Han Q, Yi Z, et al. NF-kappaB and pSTAT3 synergistically drive G6PD overexpression and facilitate sensitivity to G6PD inhibition in ccRCC. Cancer cell international. 2020; 20: 483.

22. Scannevin RH, Alexander R, Haarlander TM, Burke SL, Singer M, Huo C, et al. Discovery of a highly selective chemical inhibitor of matrix metalloproteinase-9 (MMP-9) that allosterically inhibits zymogen activation. The Journal of biological chemistry. 2017; 292: 17963-74.

23. Boon L, Ugarte-Berzal E, Martens E, Fiten P, Vandooren J, Janssens R, et al. Citrullination as a novel posttranslational modification of matrix metalloproteinases. Matrix biology : journal of the International Society for Matrix Biology. 2021; 95: 68-83.

24. Chinello C, Cazzaniga M, De Sio G, Smith AJ, Grasso A, Rocco B, et al. Tumor size, stage and grade alterations of urinary peptidome in RCC. Journal of translational medicine. 2015; 13: 332

25. Li Z, Liu J, Zhang X, Fang L, Zhang C, Zhang Z, et al. Prognostic Significance of Cyclin D1 Expression in Renal Cell Carcinoma: a Systematic Review and Meta-analysis. Pathology oncology research : POR. 2019.

26. Milioli H, Alexandrou S, Lim E, Caldon CE. Cyclins E1 and E2 in ER+ breast cancer: prospects as biomarkers and therapeutic targets. Endocrine-related cancer. 2020.

27. Chan AM, Enwere E, McIntyre JB, Wilson $\mathrm{H}$, Nwaroh C, Wiebe N, et al. Combined CCNE1 high-level amplification and overexpression is associated with unfavourable outcome in tubo-ovarian high-grade serous carcinoma. The journal of pathology Clinical research. 2020.

28. Wei R, Thanindratarn P, Dean DC, Hornicek FJ, Guo W, Duan Z. Cyclin E1 is a prognostic biomarker and potential therapeutic target in osteosarcoma. Journal of orthopaedic research : official publication of the Orthopaedic Research Society. 2020; 38: 1952-64.

29. Yang SD, Sun RC, Mu HJ, Xu ZQ, Zhou ZY. The expression and clinical significance of TGF-beta1 and MMP2 in human renal clear cell carcinoma. International journal of surgical pathology. 2010; 18: 85-93.

30. Guo Y, Xiao P, Lei S, Deng F, Xiao GG, Liu Y, et al. How is mRNA expression predictive for protein expression? A correlation study on human circulating monocytes. Acta biochimica et biophysica Sinica. 2008; 40: 426-36.

31. Joyner DE, Damron TA, Aboulafia A, Bokor W, Bastar JD, Randall RL. Heterogeneous expression of melanoma antigen (hMAGE) mRNA in mesenchymal neoplasia. Tissue antigens. 2006; 68: 19-27.

32. Powell H, Kitteringham NR, Pirmohamed M, Smith DA, Park BK. Expression of cytochrome P4502E1 in human liver: assessment by mRNA, genotype and phenotype. Pharmacogenetics. 1998; 8: 411-21.

33. MacLean C, Moenning U, Reichel A, Fricker G. Closing the gaps: a full scan of the intestinal expression of p-glycoprotein, breast cancer resistance protein, and multidrug resistance-associated protein 2 in male and female rats. Drug metabolism and disposition: the biological fate of chemicals. 2008; 36: 1249-54.

34. Bauernfeind $\mathrm{AL}$, Babbitt CC. The predictive nature of transcript expression levels on protein expression in adult human brain. BMC genomics. 2017; 18: 322.

35. Rhee SG. Cell signaling. $\mathrm{H} 2 \mathrm{O} 2$, a necessary evil for cell signaling. Science. 2006; 312: 1882-3.

36. Pelicano H, Carney D, Huang P. ROS stress in cancer cells and therapeutic implications. Drug resistance updates : reviews and commentaries in antimicrobial and anticancer chemotherapy 2004; 7: 97-110.

37. Leve F, Peres-Moreira RJ, Binato R, Abdelhay E, Morgado-Diaz JA. LPA Induces Colon Cancer Cell Proliferation through a Cooperation between the ROCK and STAT-3 Pathways. PloS one. 2015; 10: e0139094.

38. Yeh CB, Hsieh MJ, Hsieh YH, Chien MH, Chiou HL, Yang SF. Correction: Antimetastatic Effects of Norcantharidin on Hepatocellular Carcinoma by Transcriptional Inhibition of MMP-9 through Modulation of NF-kB Activity. PloS one. 2017; 12: e0171900.

39. Sogawa C, Eguchi T, Tran MT, Ishige M, Trin K, Okusha Y, et al. Antiparkinson Drug Benztropine Suppresses Tumor Growth, Circulating Tumor Cells, and Metastasis by Acting on SLC6A3/DAT and Reducing STAT3. Cancers. 2020; 12

40. Xiang Y, Li JP, Guo W, Wang DQ, Yao A, Zhang HM, et al. Novel interactions between ERalpha-36 and STAT3 mediate breast cancer cell migration. Cell communication and signaling : CCS. 2019; 17: 93.

41. Zhang $Q$, Yang Z, Han Q, Bai H, Wang Y, Yi X, et al. G6PD promotes renal cell carcinoma proliferation through positive feedback regulation of p-STAT3. Oncotarget. 2017; 8: 109043-60.

42. Mori K, Uchida T, Yoshie T, Mizote Y, Ishikawa F, Katsuyama M, et al. A mitochondrial ROS pathway controls matrix metalloproteinase 9 levels and invasive properties in RAS-activated cancer cells. The FEBS journal. 2019; 286: 459-78

43. Jiang P, Du W, Wang X, Mancuso A, Gao X, Wu M, et al. p53 regulates biosynthesis through direct inactivation of glucose-6-phosphate dehydrogenase. Nature cell biology. 2011; 13: 310-6.

44. Yang HC, Yu H, Liu YC, Chen TL, Stern A, Lo SJ, et al. IDH-1 deficiency induces growth defects and metabolic alterations in GSPD-1-deficient Caenorhabditis elegans. Journal of molecular medicine. 2019; 97: 385-96.

45. Lamonte G, Tang X, Chen JL, Wu J, Ding CK, Keenan MM, et al. Acidosis induces reprogramming of cellular metabolism to mitigate oxidative stress. Cancer \& metabolism. 2013; 1: 23. 